\title{
Revolution of Our Time: \\ Surveillance and materiality in the Hong Kong 2019 protests
}

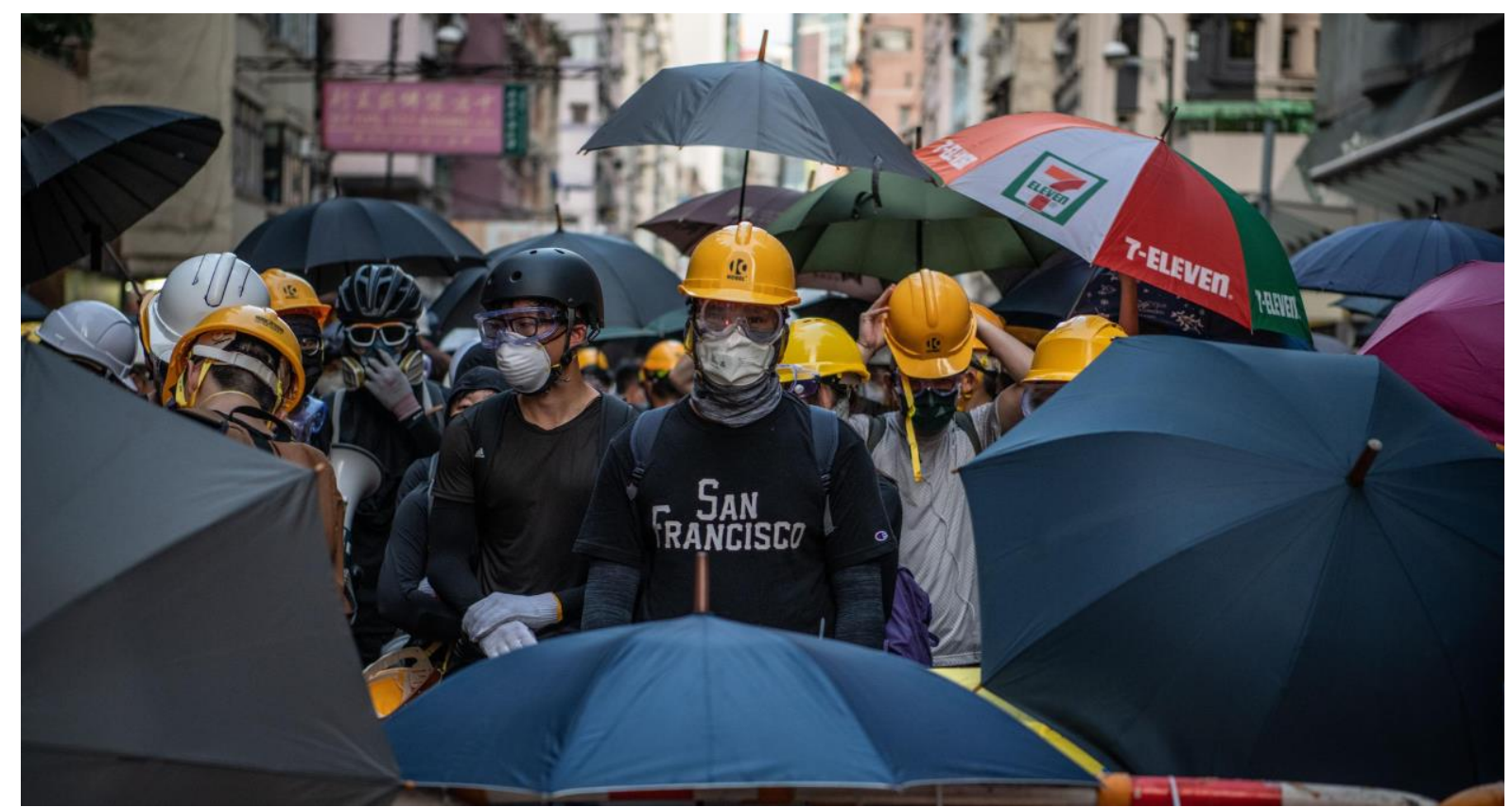

Course: Advanced Knowlegde, Organization and Politics - ASOK15101U

Lecturer: Mathias Wullum Nielsen

Course Coordinator: Charlotte Baarts

Examination number:

Emil Bakkensen Johansen: 21

Janus Emil Mariager: $\quad 16$

Victor Kalsen Nissen: $\quad 17$

Amount of characters (including spaces): 83,869 


\section{Contents}

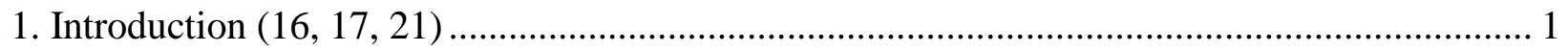

1.2 Dimensions of Knowledge, Organization and Politics (16) …………............................. 2

1.3 Contextualization: The 2019 Hong Kong Protests (17)....................................................... 2

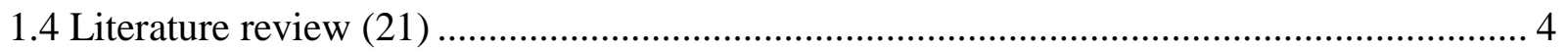

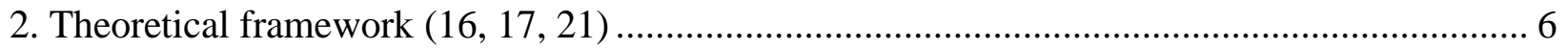

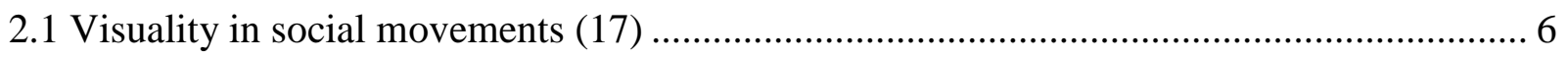

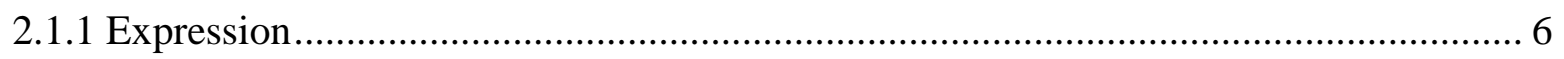

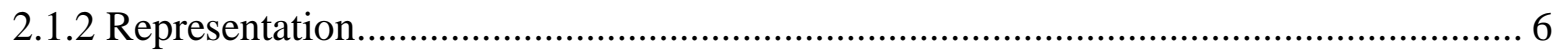

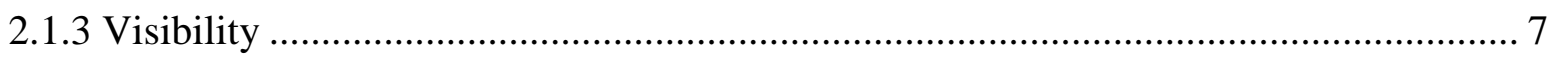

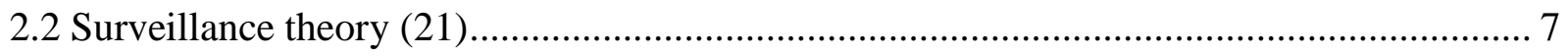

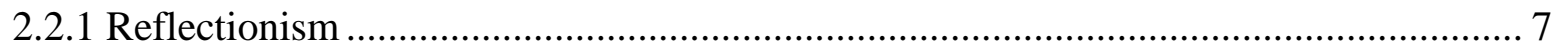

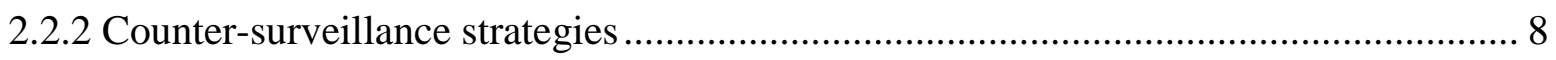

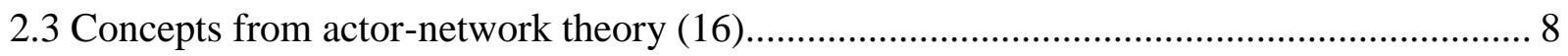

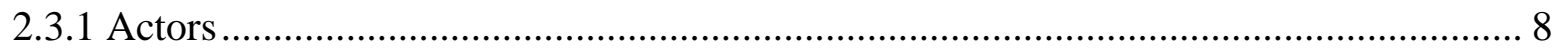

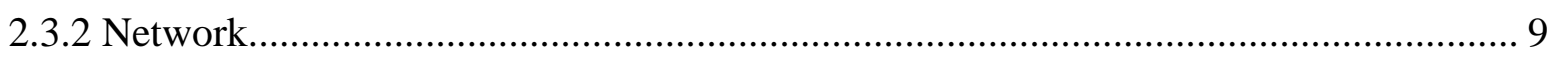

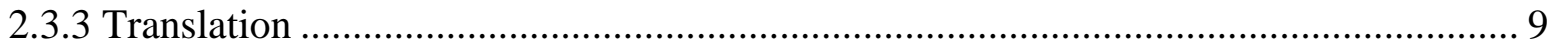

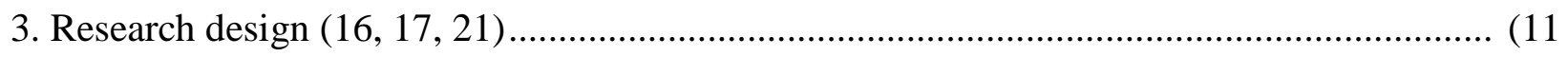

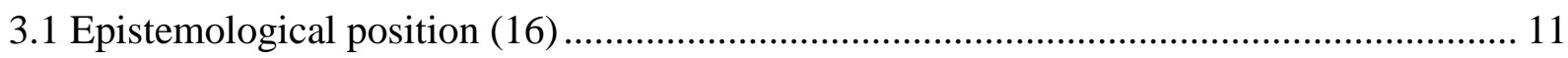

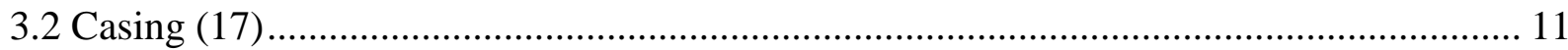

3.3 Methodological approach (21) ............................................................................... 12

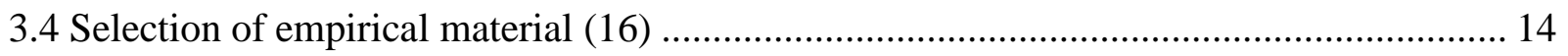

3.5 Analytical strategy (17).............................................................................................. 15

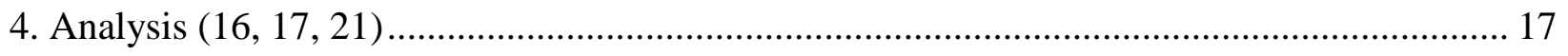

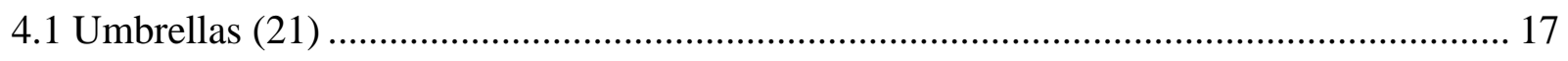

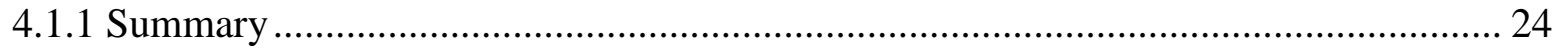

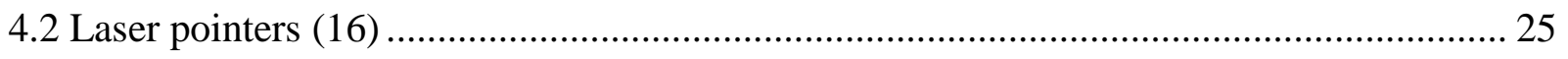

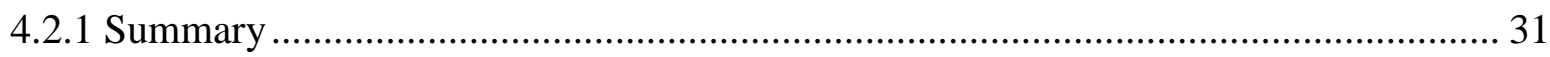

4.3 Spray paint (17)

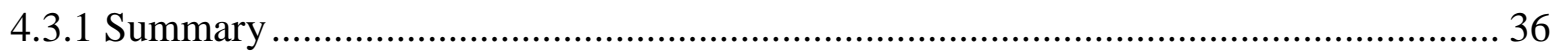




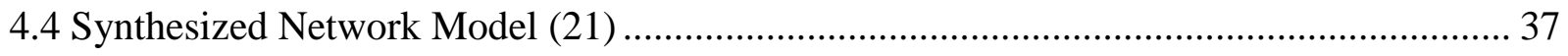

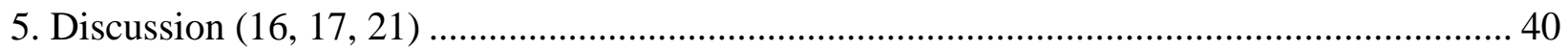

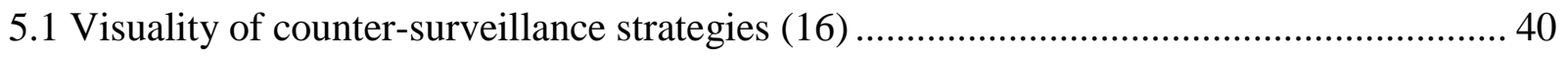

5.1.1 Counter-surveillance as a problematization tactic ............................................. 40

5.1.2 Counter-surveillance as visual expression .......................................................... 41

5.1.3 Counter-surveillance as a visual marker ........................................................ 42

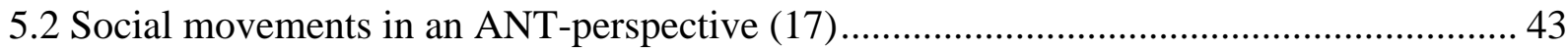

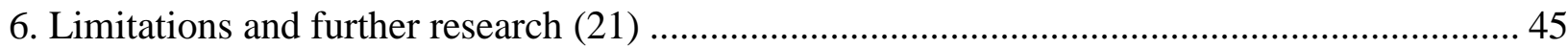

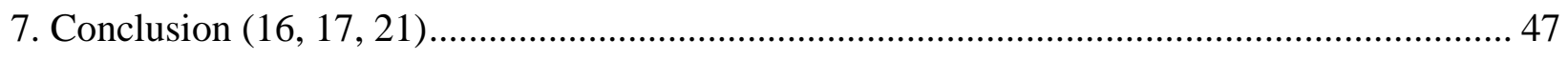

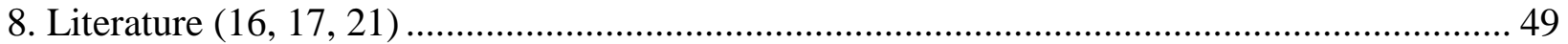




\section{Introduction}

On February 12, 2019, the Hong Kong government proposed a legal change that would allow transfer of certain criminal suspects to mainland China. This proposition ignited demonstrations initially against the proposition itself, and later against the Hong Kong government and Chief Executive, Carrie Lam. Since then, millions of protesters have been demonstrating for the political independence of Hong Kong (Leigh et al. 2019-08-14). During these demonstrations, the use of objects and technologies by protesters in their confrontation with local authorities and surveillance devices have been especially highlighted by news media (Bostock 2019-08-01; Dana 2019-11-13, Novak 2019-10-04).

We observe that the protests in Hong Kong is an example of a social movement, where material circumstances influence how the movement are organized. This contrasts with thinking of social movements in merely social terms. It is not only an ideological struggle, but in the case of Hong Kong, also a technological struggle. Through an actor-network analysis, we will examine how the presence of surveillance influences the central human and non-human actors of the protest.

Mobilization of non-human actors in social movements is an understudied area in the current literature on political mobilization and civil disobedience, and also the implications of protesting within one of the most surveilled cities in the world (Bischoff 2019-08-15). The reason could be that the Hong Kong protests are arguably one of the first times protests occur within such a context, while being highly documented in mass media.

To gain insight into the materiality in the Hong Kong protests, we deploy a theoretical and methodological approach based on a synthesis between actor-network theory and visual sociology. Our empirical foundation for this analysis is 83 photographs that serve as second-hand insight into the protest situation.

Through this qualitative analysis, we will investigate the role of non-human actors in the Hong Kong protests. Also, whether the context of protesting in a surveillance state influence the way the networks of the protests are organized. The analytical results point to relations of surveillance and counter-surveillance strategies as central connections between actors in the protest networks. In our discussion, we therefore reflect on how these strategies functions as visual markers for the social movement, and the implications our findings have for theoretical understandings of social movements.

To guide the direction of our project, we have developed the following research question summarizing the reflections above:

- How do certain technologies and objects become central actors in networks of protests through chains of translations in the Hong Kong 2019 protests? 
We understand the concepts actor, network and translation in line with actor-network theory (ANT), which we will explain further in the both the theoretical and the methodological sections. By technologies and objects, we refer to non-human actors present in the protest situation such as security cameras and umbrellas.

\subsection{Dimensions of Knowledge, Organization and Politics}

In the following section, we will state how the Hong Kong protests, our ANT analysis, and the concept of visuality relate to the dimensions of knowledge, organization and politics.

The dimension of knowledge is reflected in our project through our ANT approach. We investigate how knowledge is situated in networks of human and non-human actors, and how these actors are translated through connectedness in the network. Knowledge is thereby a combination of form and matter, and cannot be separated from its material aspects (Latour 2000). Furthermore, we investigate how visuality plays a role in producing shorthand knowledge in the protest networks. An example of this from the Umbrella-movement in 2014, is how the umbrella translates from a practical tool into a visual marker for the social movement (Chan 2015:5). In this way, the umbrella became an abbreviation, or a shorthand, for a bigger repertoire of meaning and knowledge.

In relation to visuality, the dimension of organization is relevant, when non-human actors that are central in the protest networks, translates into visual markers that function as organizational fixpoints for the social movement. An example of this in our project is how laser pointers translate into a tool for visualizing a collective. It is important to emphasize that we are not investigating formal organizations, but rather how spontaneous organizations form on 'the streets' of Hong Kong. Paraphrasing one of Latour's ANT slogans "No groups, only group formation" (Latour 2005:27), we could say: No organizations, only organizing.

Obviously, we are looking into a social movement, which is inherently politically oriented. In this project the political dimension becomes prevalent in our discussion, where we reflect on how the issue, which is a central element of any social movement, is formulated by the visual markers used by protesters. This means that when protesters use spray paint on a security camera, it is not just an act of vandalism, but a political statement against China's influence on Hong Kong. To conclude, we view political mobilization in terms of the structuring of actors as visual markers in networks based on the formulation of issues.

\subsection{Contextualization: The 2019 Hong Kong Protests}

To get a better understanding of our case, we will do a brief contextualization of the unrest in Hong Kong in the following section.

On June 16, 2019, as many as two million people took to the streets of Hong Kong (Leal 2019-0816). The event was the largest political mobilization in the history of Hong Kong, and what followed was months of violent protests that are still ongoing as of this writing. Although the initial 
spark that ignited the protests was the proposal of a legal change that would allow for extradition from Hong Kong to mainland China, the underlying reasons for the unrest has been fuming for decades (Anon 2019-10-23; Tai 2019).

In 1984, the People's Republic of China signed an agreement, where the United Kingdom agreed to hand over control of Hong Kong, with the promise from China that the area would enjoy a high degree of autonomy as a Hong Kong Special Administrative Region (HKSAR). The agreement proposed the idea of 'One Country, Two Systems', where Hong Kong would formally become part of China, while remaining politically and judicially autonomous (Wong \& Mak 2019-10-30). The first democratic movement in Hong Kong can trace its origin to this period, but its first real emergence would surface as a response to the Tiananmen Square Massacre in 1989 (Tai 2019:65). Later, the cry for democracy would reappear in 2003, when over half a million people marched in protest to the proposal of a national-security law, which many worried would become a tool for quashing dissent (Tai 2019:66). At the time, this marked the largest public rally in the history of Hong Kong, which resulted in the withdrawal of the bill, and the resignation of the first chief executive of the HKSAR.

The foundation of the current unrest is mostly rooted in the Umbrella Movement from 2014 that was triggered by a student strike, objecting to China's decision to impose restrictions on the election of the Chief Executive of Hong Kong (Chan 2015:1; Kaiman 2014-09-30). After Beijing rejected the promise of free elections with universal suffrage, student activists took to the streets to occupy central intersections in the city of Hong Kong. The protests became known as the Umbrella Movement for its characteristic use of umbrellas as a symbol for non-violent protesting (Chan 2015:5). Despite ending without any substantial gains in the fight for democracy, the protest of 2014 showed that the people of Hong Hong had become much more receptive to civil disobedience as a tool for democratization (Tai 2019:68).

On February 12 2019, a bill that would allow criminal suspects to be extradited to mainland China was proposed by the Hong Kong Government (Anon 2019-10-23). Mirroring the events in 2003, the bill was criticized for allowing Hong Kongers to get exposed to the flawed justice system in China and that it would lead to further erosion of the HKSAR's judicial independence (Li 201912-13). While the bill was scaled back in the following months, criticism against the proposal continued to escalate and culminated on the 16th of June with the aforementioned largest demonstration in the history of Hong Kong (Leal 2019-08-16).

Protesters have utilized a wide variety of practices to demonstrate against the government. This includes peaceful practices, such as city-wide human chains, collective singing and long marches. Other protesters have turned to more extreme measures such as blockading the Hong Kong Airport, occupying universities and storming the Legislative Council (Wu et al. 2019-11-20). Throughout the events, protesters have turned violent by throwing bricks, bottles and fire bombs against police officers, who in return have faced the protesters with pepper spray, batons and tear gas. The 
authorities' answer to the demands of the protesters has been increasing police brutality, the activation of colonial-era emergency powers, and the use of live rounds against protesters (Leal 2019-08-16).

While the Extradition Bill that initiated the unrests was withdrawn on September 4, the protesters' demands had since grown to minimally include five specific demands (Anon 2019-11-28):

1. For the protests not to be characterised as a "riot".

2. Amnesty for arrested protesters.

3. An independent inquiry into alleged police brutality.

4. Implementation of complete universal suffrage.

5. The withdrawal of the extradition bill.

The Hong Kong Government has so far been unresponsive to the four remaining demands (Kam 2019).

\subsection{Literature review}

In the following section, we will explain how our project is related to earlier studies within the frame of social movements. Furthermore, we will explicate how an ANT perspective will provide other insights, than what is the usual focus of similar studies of particular social movements.

The literature on social movements has a long history and is rich in theoretical traditions. One branch is the classical approach, where social movements are related to politics of redistribution (Nash 2010). Later came a modern understanding of social movements, labeled new social movement theory. Here the economic and material interest of individuals were replaced by singular issues based on politics of identity (Melucci 1989).

Within these theoretical frameworks, the involvement of non-human actors in social movements is understudied, as these studies have a tendency to prioritize human actors and social mechanisms. In the case of the Hong Kong protests, however, it is evident that the presence and actions of nonhuman actors should not be glossed over, but rather become subject to inquiry. Based on our findings in this project, we will reflect on the implications for future studies of social movements in our discussion.

However, it is worth noting that the issue mentioned above has been analyzed in political mobilization literature. Several articles on the protests during the Arab spring have focused on the use of social media and its role in mobilizing citizens for protests, as well as a means to spread information globally. Another example is an article on protests in Spain, where the authors argue that Twitter was used as a counter-surveillance tool by protesters by sharing videos online through the platform (Hermida and Hernández-Santaolalla 2018). Other articles focus specifically on the role of video surveillance in regards to social protests, and how protesters react to the presence of surveillance technologies (Ullrich and Knopp 2018). One argues for example that the presence of 
video surveillance in situations of public protest in Germany changes the protest situation itself (Ullrich and Wollinger 2011).

As mentioned, we use an ANT approach in this project, while analyzing the role of surveillance and counter-surveillance technologies being mobilized in the Hong Kong protests. ANT-oriented studies on political mobilization have been conducted (Rodríguez-Giralt 2011; Rodríguez-Giralt, Marrero-Guillamón, and Milstein 2018). However, we have not identified ANT-based articles on political mobilization that focus specifically on surveillance technologies and non-human actors in public (and violent) protests, but rather articles on more civil, peaceful, and primarily environmental demonstrations (Bennett 2018).

We believe our project can contribute to this lack of focus, which we find important to illuminate, because it seems plausible that protests similar to the Hong Kong 2019 protests can occur in the future. To sum up, our project sets out not to give primacy to human actors at the outset of inquiry, but to take into account the materiality, visuality and non-human actors that are present in the protest situation. Through this approach, we argue, it is possible for social movement theory to illuminate not just what the issue of a social movement is, but also the process of issue formulation. 


\section{Theoretical framework}

In this section, we will present the theoretical framework of the research project. The framework is separated into three parts. Firstly, we will present theories and concepts from visual sociology deployed on social movements by Nicole Doerr, which will be relevant in the discussion. Secondly, we will present surveillance and especially counter-surveillance theory by first Steve Mann, and thereafter by Peter Ullrich and Philipp Knopp, which will be relevant in our analysis and the following discussion. Thirdly, we will present key concepts from actor-network theory (ANT) by Bruno Latour and Michel Callon that are central to our analysis.

\subsection{Visuality in social movements}

According to Nicole Doerr, social movements are intrinsically tied to the visual sense, both in how they are perceived by the members of the movement, and in how they act in intended or unintended ways (Doerr 2013:xi).

Social movements form as a collective actor by using and reinterpreting a preexisting imaginary to voice critique. In this context, social movements can tap into rich reservoirs of social and cultural knowledge that both marks their affiliation with a collective, and identifies their position in a social or political conflict (Doerr 2013:xiii). Continuity is established in these movements by using colors, symbols, graphic design and other visual imagery to connect different activist identities across generational and contentional divides.

\subsubsection{Expression}

For the individual activist, the self-expression of clothes and gestures acts as the primary carrier of messages to spectators. Activists in social movements use their bodies to expose and embody a deviant mindset (Doerr 2013:xiv). As an example from our research topic, we see specific clothing and accessories play a prominent visual part in the Hong Kong demonstrations. Activists can often be seen with yellow helmets, face and gas masks, black clothing, umbrellas and other characteristic objects. For our purposes, we will argue that these modes of expression, described by Doerr, can be expanded to include visually expressive actions through the use of tools, such as umbrellas, laser pointers and spray paint.

\subsubsection{Representation}

Visuals also play an important role in the representation of social movements. Doerr writes that "protests are not perceived as what they are on the ground, but what they look like in the press photos and television news images" (Doerr 2013:xiv). The meaning behind the imagery of social movements is often colored by the means of access in which recipients come into contact with the visuals. This means that mass media traditionally have had a substantial influence in the ways in which movements are perceived. This dominance is now being challenged by the emergence of online social media, where protesters now have more direct control over the representation of the 
movement (Doerr 2013:xv). In regard to the media's influence on representation, it is also important to keep in mind that viewers are not a passive audience, but are actively decoding and interpreting imagery based on their own experiences and knowledge (Doerr 2013:xiv).

\subsubsection{Visibility}

Another important part of the operation of social movements is the necessity of visibility. Protesting is always a matter of gaining visibility for social or political problems and establishing the movement as a conflict party (Doerr 2013:xv). This raises interesting points about the unequal opportunities for different movements and compatibility of the issues they are trying to raise awareness about (Doerr 2013:xvi). But more pressingly, it also shows that the visual imagery of social movements does not just orbit around matters of expression and identification, but is also a fundamental tool of spreading awareness, constituting movements as actors and the diffusion of different ideas and narratives (Doerr 2013:xv).

Overall, visuality is a factor that ties together social movements internally and externally, while also constituting the movement as an actor in society.

\subsection{Surveillance theory}

In our analysis, we find that relations of surveillance and counter-surveillance are central issues in the Hong Kong protests. To understand the role of these relations, we will employ theories on surveillance and counter-surveillance based on Steve Mann's concept of reflectionism as well as Ullrich Knopp \& Peter Ullrich's notion of counter-surveillance strategies. These concepts and understandings will be elaborated upon in this section, and will be utilized in the discussion.

\subsubsection{Reflectionism}

According to Mann, the notion of a surveillance society has moved from abstract ideas in the sphere of fictional dystopian novelties to become realized entities (Mann 2003:332). He exemplifies this by the increasingly widespread use of security cameras in public spaces. To Mann, the existence of these surveillance tactics, deployed by bureaucratic organizations such as states, creates an imbalance in power relations.

Through his work, Mann develops the idea of reflectionism as a tactic to counter this imbalance. This concept encapsulates, when subjects use technology to mirror and confront the surveillance by bureaucratic organizations (Mann 2003:333). One function of reflectionism is its ability to comment and question the very act of surveillance itself, when it is done through explicit and visible ways (Mann 2003:337). Here the goal is not necessarily to neutralize the surveillance itself, but instead to make it visible, problematize it and make it subject to public debate and scrutiny. 


\subsubsection{Counter-surveillance strategies}

Ullrich \& Knopp build further upon Mann's understanding in their analysis of protester's reactions to police video surveillance in Germany (2018). They identify six counter-surveillance strategies: consider cameras, disguise, attack, hide, sousveillance and formal ways of controlling the police, and cooperation.

In this project, the most relevant counter-surveillance strategies are attack, disguise and hide. Attack is when the protester acts in aggressive or offensive physical ways towards devices of surveillance. An example of such a strategy could be crushing the lens of a security camera. The authors argue that such a move can be framed as symbolic political action towards a surveillance state (Ullrich \& Knopp 2018:190). On the other hand, disguise as a counter-surveillance strategy is collective deployment of defensive techniques, aiming at rendering protesters non-identifiable. An example of this could be wearing similar clothes so as not to stand out, but as Ullrich \& Knopp also suggests, utilizing umbrellas as shields. The latter is one of several strategies that will be made visible through our analysis. Contrary to the disguise strategy, hide refers to predominantly legal strategies to hide from cameras on an individual level (Ullrich \& Knopp 2018:190).

\subsection{Concepts from actor-network theory}

In this section, we will present the actor-network theory (ANT) perspective. The analysis will use ANT terminology as it is formulated by Bruno Latour and Michel Callon, where the focus will be on mapping connections between actors in networks, and to show how these actors translate each other. Thus, we will now outline the following core concepts of ANT: actors, networks and translations.

\subsubsection{Actors}

The concept of actors emerges as a critique of the separation between human and non-human, culture and nature, subject and object, which is posed by Latour amongst other places in We Have Never Been Modern and alongside Callon in Unscrewing the Big Leviathan. Here, it is emphasized how this dialectic has formed social sciences since Immanuel Kant made the distinction between things-in-themselves and the subject precepting it (Latour 1993:57; Callon \& Latour 1981:301).

Instead of this distinction, we should understand all actors once divided into either culture or nature, under the same category called actors, which can be both human and non-human actors. At the essence of an actor, lies the notion that actors possess agency. Or as Callon puts it: "Thus it acts: it is an actor" (Callon 1998:140).

The task of the researcher becomes to follow those actors and map out their relations, as Latour formulates it in Reassembling the Social: 
Using a slogan from ANT, you have to 'follow the actors themselves', that is try to catch up with their often wild innovations in order to learn from them what the collective existence has become in their hands, which methods they have elaborated to make it fit together, which accounts could best define the new associations that they have been forced to establish (Latour 2005:12).

Equally important when defining the essence of an actor in ANT, lies in the heterogeneity of the actors, which means that they can do and be anything (Callon 1998:136; Callon \& Latour 1981: 292). It is exactly these heterogeneous associations that should be followed (Latour 2005:8). The actor's collective existence, the methods making them fit together, and the associations that they establish, can be summed up in the other central and closely related concept of ANT: network.

\subsubsection{Network}

First and foremost, the network is the place in which the actors are situated and connected to each other. In that way, actors connected to each other form a network, which can also act as an actor in another larger network (Callon 1998:142-143).

For Latour, a network is a concept more than a thing 'out there', meaning that it is constructed by the researcher. Furthermore, a network always consists of actors, why it is called a actor-network (Latour 2005:132). An important thing is that all these actors must act to be a part of the network, or as Latour formulates it:

To put it very simply: A good ANT account is a narrative or a description or a proposition where all the actors do something and don't just sit there (Latour 2005:128).

So, to trace a network, one must add every relevant and acting actor to it. This means that the amount of intermediaries and especially translations increases, which strengthens the existences of the network (Latour 2005:133).

\subsubsection{Translation}

Another central concept within the theoretical framework is the notion of change, which in the terminology of ANT is defined as translation. Callon \& Latour define it as the following:

By translation we understand all the negotiations, intrigues, calculations, act of persuasion and violence, thanks to which an actor or force takes, or causes to be conferred on itself, authority to speak or act on behalf of another actor or force (Callon \& Latour 1981:279).

Thus, translation is the process in which an actor, which can both be human and non-human, changes due to its connection to one or more actors within a network. This means that actor A, translates actor B. For a translation to undergo, "it involves a translator, something that is translated, and a medium in which that translation is inscribed" (Callon 1998:143). 
In relation to this, a crucial criterion of understanding a scientific fact as being true, is the notion that these so-called chains of translations cannot be broken (Latour 1999:69). This means that the relations between different human and non-human actors must remain within the network, which means that actors not being connected to other actors are disconnected from the particular network. 


\section{Research design}

In this section the research design for this project will be presented. First the epistemological position of this project will be elaborated upon. This is based on ANT. Following this we will describe our process of casing the Hong Kong protests in this project. Additionally, we will describe the methodological approach, which is based on Douglas Harper's thoughts on visual ethnography. Lastly, we will present our process for selection of empirical material as well as our analytical strategy for studying the photographs.

\subsection{Epistemological position}

This project epistemological standpoint is within the tradition of actor-network theory (ANT), which is closely related to Science and Technology Studies (STS), and especially the works of Bruno Latour and Michel Callon, which are the ANT related theorists that our theoretical framework is built upon.

According to Latour, the core aspect of ANT inspired research practice within sociology is the transition from "sociology of the social" to a "sociology of associations", where the focus or the object of analysis shifts from being on the social itself to being on relations between actors that are social due to their connectedness, but not from a somewhat essential sociality (Latour 2005:9). In our analysis this will be shown by mapping out the network of specific protest situations based on photographs, and thereafter explain how a specific actor is translated as a product of its relations to other actors. An example is the laser pointer that in one relation can be understood as a tool to blind policemen and in another relation be translated into a tool for visualizing a collective.

Another important aspect of ANT is that it contains a degree of constructivism but emphasizes how and why this is not to be mistaken with social constructivism. A constructed fact means that "we account for the solid objective reality by mobilizing various entities whose assemblage could fail", whereas social constructivism a priori constitutes the idea that reality is built upon something social (Latour 2005:91). The consequence of this in our project is that we consider the Hong Kong protest as constructed through networks of both human and non-human actors, and we thereby reject the idea of it being exclusively socially constructed, which could have been the case if we for example analyzed discourse. Instead we look at specific technologies and objects throughout the analysis and focus on how they are connected to protesters in specific situations.

\subsection{Casing}

In this project we have chosen to take on the Hong Kong 2019 protests as a case. In this section we will go through our casing process.

We have deployed Ragin's “casing” model, which consists of six steps (Ragin 1992:221-223). First, we established the social phenomenon that we wanted to direct our project towards, which was political mobilization. Then we narrowed our theoretical focus towards social protests in light 
of new technologies and mobilization in surveillance states - more specifically the role of nonhuman actors in these situations. To narrow our perspective further, we chose to investigate people fighting for freedom rights, specifically the Hong Kong protesters. To investigate the case of the Hong Kong protesters, we then limited our empirical lens towards photographs of the protests.

Ragin states that "usually a problematic relation between theory and data is involved when a case is declared" (Ragin 1992:218). Our case is no exception, as our main interest in this project is the form of protesting that takes place in Hong Kong, which the current literature on social movement has not highlighted thoroughly. In other words, it is an anomaly that poses as a problem to the existing understandings of social movements (Pedersen 2011). The case can then be understood as an operational tool to study the importance of non-human actors in social movements.

A common misunderstanding about case studies is that they do not contribute to scientific development, because they cannot be used to create formally generalizable claims (Flyvbjerg 2006:221). Therefore, we would like to emphasize that it is not our goal to formulate a universal law of social movements. Rather, in respect to our ANT-based epistemological position, we produce situated explanations. Consequently, we do not claim that our findings can be used to predict the outcome of future protests in similar circumstances, but that the knowledge generated may be transferable, even though it is not formally generalizable (Denzin \& Lincoln 2012:177).

\subsection{Methodological approach}

In the following we will describe the methodological approach for this project and specifically our use of visual ethnography. This will be elaborated upon on the basis of Howard Becker's and Douglas Harper's methodological reflections. Before that we will contextualize the utility of alternative methodological approaches as well as less classical empirical methods.

According to Mike Savage and Roger Burrows, a crisis within empirical sociological research has emerged in the age of knowing capitalism. Today, the methodological superiority of sociologists has decreased in the light of circuits of information's embeddedness in multiple information technologies as well as its increasingly central part of private corporations (Savage \& Burrows 2007:4). So what should sociologists do regarding empirical material and methodological approach?

This call [...] asks for greater reflection on how sociologists can best relate to the proliferation of social data gathered by others, which we currently largely ignore (Savage \& Burrows 2007:13).

As a consequence of this development, sociologists should turn to different types of empirical material and also data gathered by others, which is exactly what we do in this project, where we build our analysis on photographical material conducted by photographers affiliated with Reuters. 
The call for sociologists to widen their empirical scope is also echoed by Howard Becker, who proposes a visual sociology (Becker 1995). In doing so, he questions sociologists' neglection of photography as an empirical source. This aversion is based upon a view of photographs as unscientific, because they are neither objective or neutral accounts of social reality and easily manipulated (Becker 1995:7-8). While this might be true, according to Becker, the potential lack of objectivity is also present within more traditional methods of sociological inquiry, thus rendering the critique of photographical sources moot (Becker 1995:10). What distinguishes visual sociology from that of documentary and journalistic photographs is the way context is provided (Becker 1995:10). What is essential to a sociological approach to photographs is to make the context and sociological analysis explicit, which can provide the picture added evidentiary value and meaning (Becker 1995:12).

One of the sociologists that has taken on the mantle of a visual methodological approach is Douglas Harper. According to Harper, photographic material as documentary are characterized by several attributes: verisimilitude, relevance and sympathy (Harper 2012:18). These terms can help us understand for what purposes we can use photographic material for our analysis.

The verisimilitude of the photographs means that they show something that existed at a given point in time and location. For this project, this means that when we look at our material, we see momentary snapshots of actors, their practices, the tools and tricks they utilize and their immediate relation to each other. On the other hand, it is not possible from this material to get any meaningful insights into the universality of these instances, or neither what is the personal significance for the actors involved.

The last two attributes are 'relevance' and 'sympathy'. Relevance is the requirement of the documentary image to be a medium that addresses political or social issues, whereas sympathy entails that the documentary image is expected to engage the viewer (Harper 2012:19). The notion of relevance could be a potential problem when gathering empirical material, since there is a risk that certain aspects of the Hong Kong protests have not been documented by our sources, due to the limitations caused by the ambition of being relevant. The chances of this becoming a problem in our project are slim, since our primary focus is a major political struggle with global attention, but it is a relevant point to keep in mind. The sympathy aspect poses another potential challenge for our data selection, because it means that the available photojournalistic images might not include less extreme or banal aspects of the events in Hong Kong. This means that our material will likely be skewed towards the more extreme practices and that certain modes of action might be underrepresented. Because we are not trying to make points about the universality of our findings, this should not be a detriment to the validity of our analytical points.

As Harper states, "visual ethnography is an effort to understand culture by making it visible" (2012:11). Cultural and social practices can be documented as visual evidence where it is possible to showcase different modes of practice and interactions. Actors, themes of action, its locality and 
factors influencing social users are usually intertwined and photographic material can capture these interconnections in a snapshot of a moment (Harper 2012:74).

Since we aim to analyze networks of actors, this density of information makes visual material a useful resource because it can capture the multiple elements that makes up an actor-network and the immediate relations in it. On the other hand, a disadvantage to our reliance on photographic material, is that it is difficult to make any substantial claims about the larger network at play since each photograph is situated in a specific time and place. Gaining a more overall understanding of the network, it would then be necessary to complement the visual material with other types of data (Harper 2012:58). Since our goal is not to map out the entire network, but instead describe microinteractions in specific situations, these disadvantages will not be a detriment to the findings of our analysis.

\subsection{Selection of empirical material}

In the following we will present the criteria for the selection of empirical material in this project.

We wanted to establish a protocol of selection criteria before beginning our collection of photographs, which could provide us with a systematic approach. Initially, we wanted to find a fitting source for collecting photographs (Altheide \& Schneider 2012:39). We chose the photographical archive of the news agency Reuters as our source. This was done for two reasons. Firstly, from a practical standpoint, as Reuters provided an accessible archive that contained a large amount of pictures, coupled with a search engine that allowed us to establish fixed criteria for our selection process. Secondly, the photographs from Reuters were taken with the intention of getting sold to other media outlets, rather than with a specific agenda in mind.

Our search string on Reuters' photographical archive was "Hong Kong protests". We limited our search to a period of four months from June 1 to October 1, 2019 and to photographs taken specifically in Hong Kong. This choice was based on when the protests escalated (Leigh et al. 2019-08-14). By using these criteria, we limited the amount of search results, and ended up with 5,554 photographs.

Our goal was then to do a preliminary sorting of these pictures. To do this, we made a list of four criteria based on the scope of our research question:

1. Non-human actors must be present in the situation.

2. Non-human actors must have a functional or visual meaning.

3. Non-human actors must engage in an interaction (with human actors (protesters)).

4. Focus is on protesting activities. 
The preliminary sorting based on these four criteria resulted in 83 unique photographs, which lived up to the criteria of the above.

Afterwards, we coded and categorized these 83 photographs. This was done by giving each photograph 'tags' based on the non-human actors that were shown. This resulted in 19 different tags. Examples of these tags are slingshot, tear gas canister and mobile phones. Then we looked at which tags were the most prominent in our coding, and based on this, made decisions for which actors should be subject to analysis in our project: umbrellas, laser pointers and spray paint.

\subsection{Analytical strategy}

In the following section, we will emphasize the strengths of using photographical material within an ANT scope, by showing examples of how Latour has done so. Afterwards, we will explain our qualitative analytical strategy, and show how it is based on ANT as well.

Using photographs as our empirical material is neither special nor original, when conducting research within an ANT framework. An example of this is Latour's fieldwork in the Amazon, which is described in Pandora's Hope. In the book, Latour uses his camera to capture concrete situations of for example a soil samples journey - and translation - from the jungle to the datasheet after being examined in the laboratory (Latour 2000). The photographs are a useful tool for Latour to understand how scientific knowledge is produced and constructed in practice.

As we also look at practice, how the protesters use specific objects, or in ANT terminology, how the relation between human and non-human actors forms the network, we consider photographical material as an informative approach to gain access to protest networks in Hong Kong.

When subscribing to an ANT perspective, the first point of the analytical strategy is to "to follow the actors themselves" (Latour 2005:12). The question is then, how to do it in our particular project. Another of Latour's work including photographical material is the project Paris: Invisible City, where the ambition was to map out and illuminate some of the many invisible networks of the French capital. In the following quote, we see how idea of the network can be investigated through photography:

Something else orders and locates, gathers and situates, binds and distinguishes, sets the pace and the rhythm, but that something no longer has the shape of a Society and must be followed, step by step, by other methods - through photography, perhaps, or rather through series of photographs that we would need to learn to read continuously (Latour \& Hermant 2006:5).

Rather a guideline than rule, we can use a series of photographs to understand not society itself, but to understand specific situations. That something else will thereby become the complex network of human and non-human actors, as we attempt to map in the forthcoming analysis. 
Using photographs will provide us insights and thereby help us map out the network in the same manner as Latour has done in other ANT studies. Yet, there are still particularly one significant difference between this project and the one it is inspired by: Latour shot the photographs himself; we did not. Instead our photographical material relies on the work of others.

In our analysis, we will construct models for each photograph consisting of actors and their relations that forms the network. We limit our mapping of networks to what can be seen in the photograph itself, thus not including actors and relations that we could only assume to exist outside the frame. We are also aware that there in each photograph is a camera and photographer present to make the photograph possible, but as these are not visible, we will not include them in the models. Yet in some of the photographs, where they actually are visible, we do not include them, as they, visible or not, are present in all of them. For the sake of simplicity, we have not mapped out each actor in every model, but rather pooled them into a maximum of three illustrated actors to show that there is more than one. If we for example see ten protesters using laser pointers in the same manner, we will only map out three of them in the network model.

After we have mapped out the network of each of the photographs in the analysis, we will map out a summarizing network model for each actor - umbrellas, laser pointers and spray paint - that is based on each of the smaller networks constructed from the photographs. In the end, we will construct are synthesized network consisting of the summarizing network models. This is done with the purpose of comparing different translations of actors in different relations. Our claim is not that this synthesized network model is a complete map of the protest network that shows all connections and translations of the actors but should only be interpreted as an analytic tool to sum up our findings. 


\section{Analysis}

In the following pages, we will present the results of our analysis that is divided into three sections. In the first section, we will analyze photographs that contain umbrellas, in the second section laser pointers, and in the third section spray paint. For each photograph, we will construct a network model, and for each section we will summarize our findings in a summarized network model that shows the different translations each actor goes through. Lastly, we will present the synthesized network model.

\subsection{Umbrellas}

In this section of the analysis, we will look into the networks of one of the non-human actors identified in the material: umbrellas. Through network analysis of four photographs, where umbrellas are used, we will map out their relations, and in that way show how the umbrellas are used in different ways. Or said in the terminology of ANT: how umbrellas are translated as a result of their relations within a network of a protest situation. We will conclude the section by summarizing our findings.

Here follows the analysis of the first photograph.

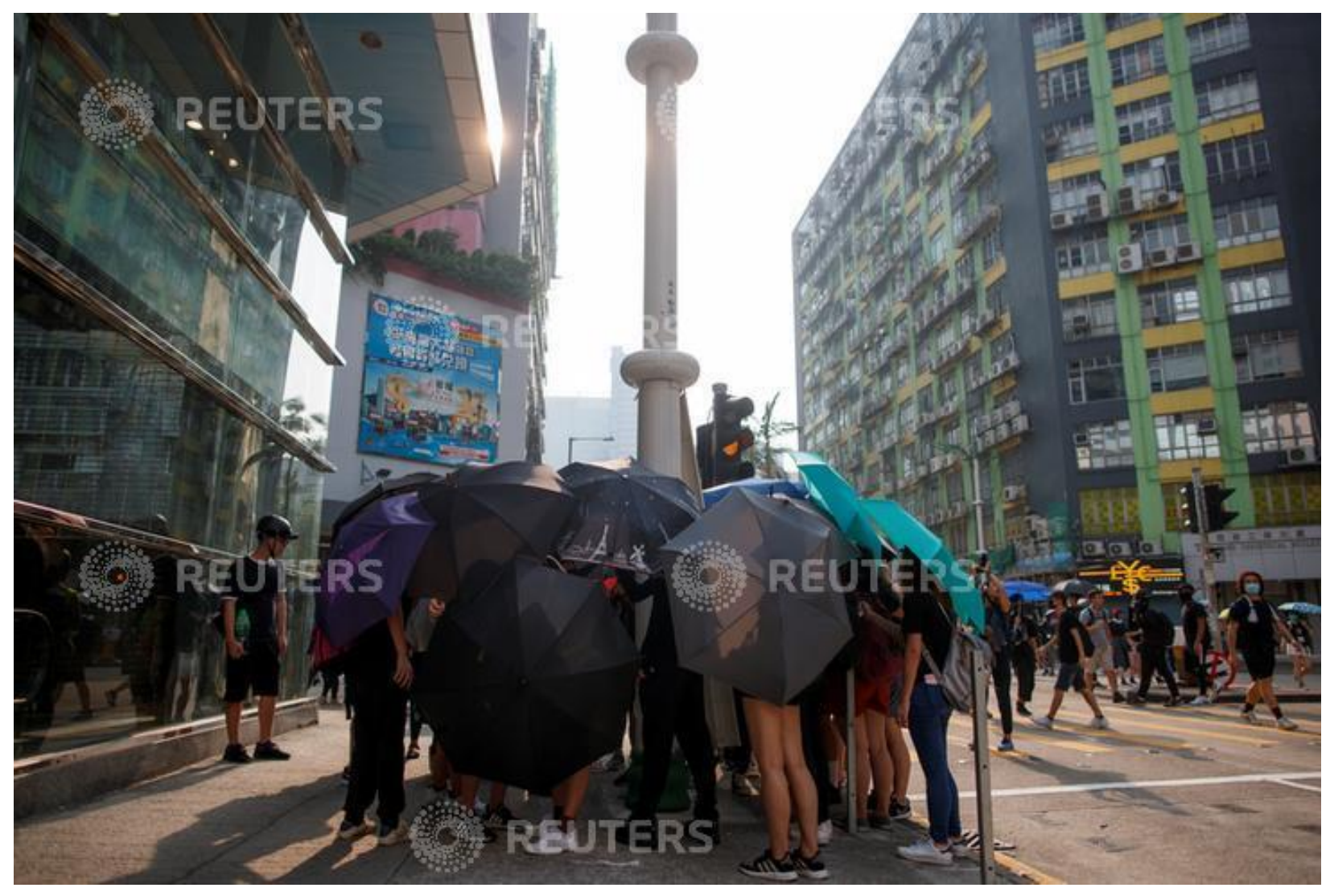


Photograph 1.1. Description from Reuters: "Protesters use umbrellas to shield themselves as people damage smart lamppost in Ngau Tau Kok in Hong Kong, China, August 24, 2019."

According to Reuters this photograph portrays protesters using umbrellas to shield themselves. The lamppost in the middle of the photograph is a so-called smart lamppost, which have been suspected by protesters as being tools of surveillance used by the Hong Kong government, due to its many sensors (Livni 2019-08-24). According to the local authorities however, these smart lampposts only exist to collect data on traffic, weather, and air quality, and will not be used to collect facial or personal data (Yeo 2019-07-19).

In the photograph we can identify three primary types of actors. Firstly, we observe several protesters gathered around the lamppost in order to sabotage it. Secondly, we identify for each protester an umbrella, which hide their faces and torso as well as the saboteurs of the smart lamppost. Thirdly, in the midst of it all, a smart lamppost.

This observation can be mapped out as the following figure 1:

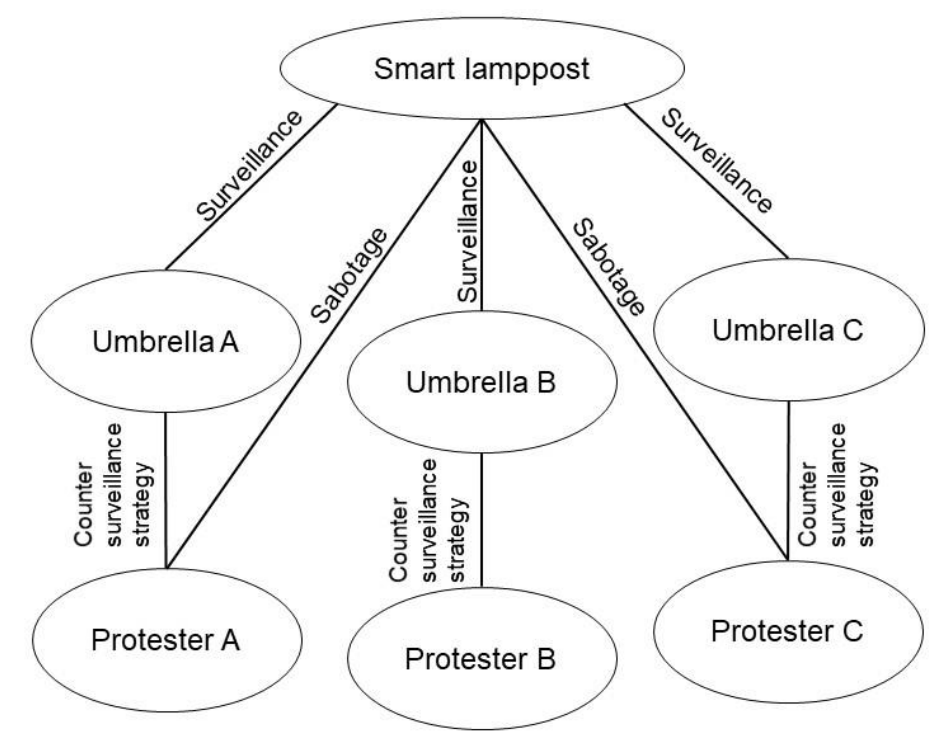

Figure 1.1. Network analysis of photograph 1.1.

The protesters are linked with the umbrellas through their use in counter-surveillance strategies. Here the strategy deployed is the move of disguise, as the protesters collectively disguise the saboteurs through the umbrella shield wall. The umbrellas, on the other hand, are connected to the lamppost by the means of (possible) video-surveillance. Finally, the protesters are linked to the lamppost by their sabotage effort. 


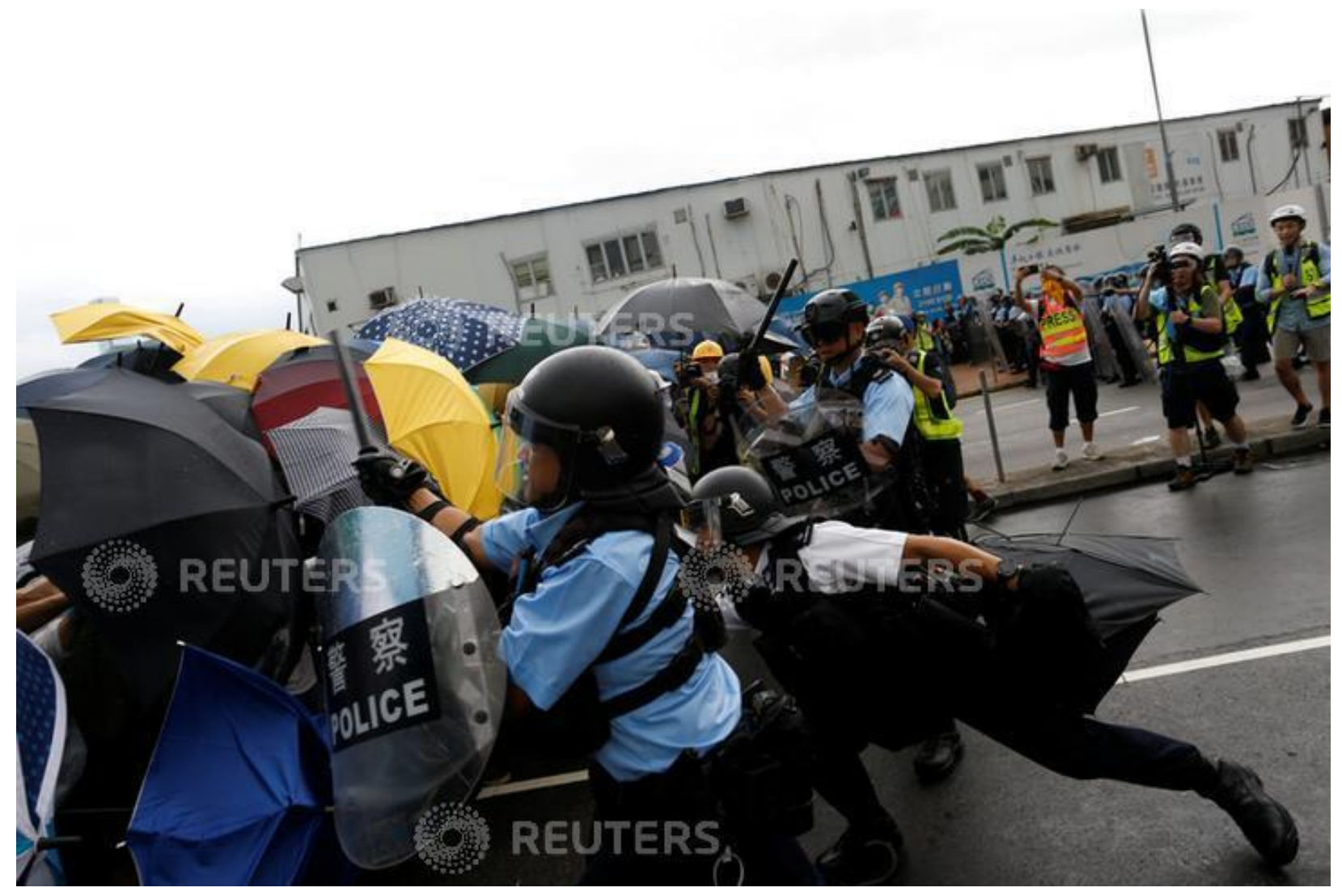

Photograph 1.2. Description from Reuters: "Riot police try to disperse protesters near a flag raising ceremony for the anniversary of Hong Kong handover to China in Hong Kong, China July 1, 2019.”

In photograph 1.2, we see another example of protesters using umbrellas as a shield wall. However, the wall in this case is used for the purpose of avoiding violent confrontations with the police.

In the photograph, we identify four primary actors. Firstly, we identify the riot police. Secondly, the riot equipment in their hands. Thirdly, the shield wall of umbrellas. Fourthly, the protesters themselves. 


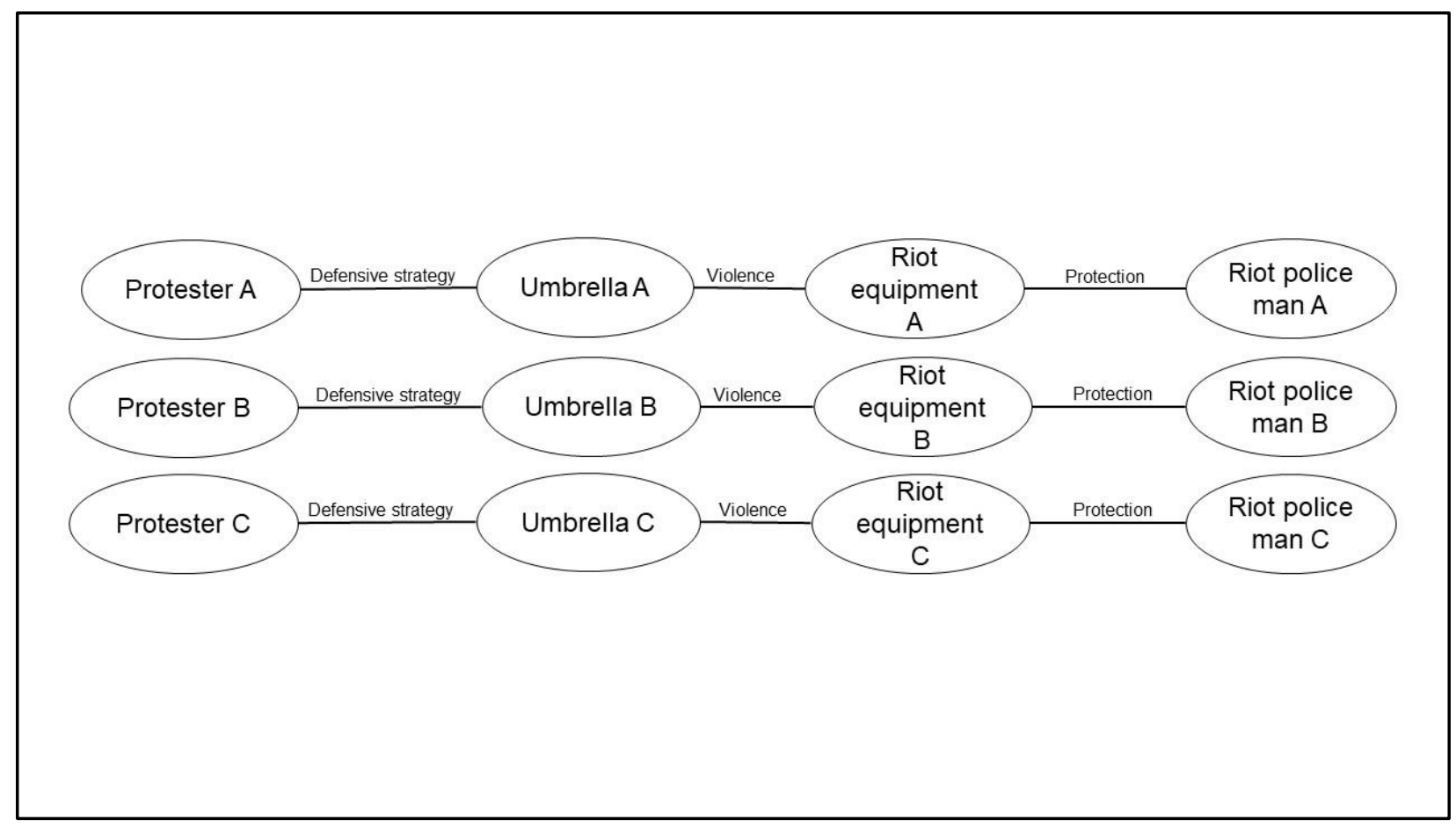

Figure 1.2. Network analysis of photograph 1.2

In this case, the protesters are linked with the umbrellas by means of defensive strategies, whereas the umbrellas are linked with the riot equipment in terms of violence. Conversely, the riot equipment is connected to the policemen for protection. 


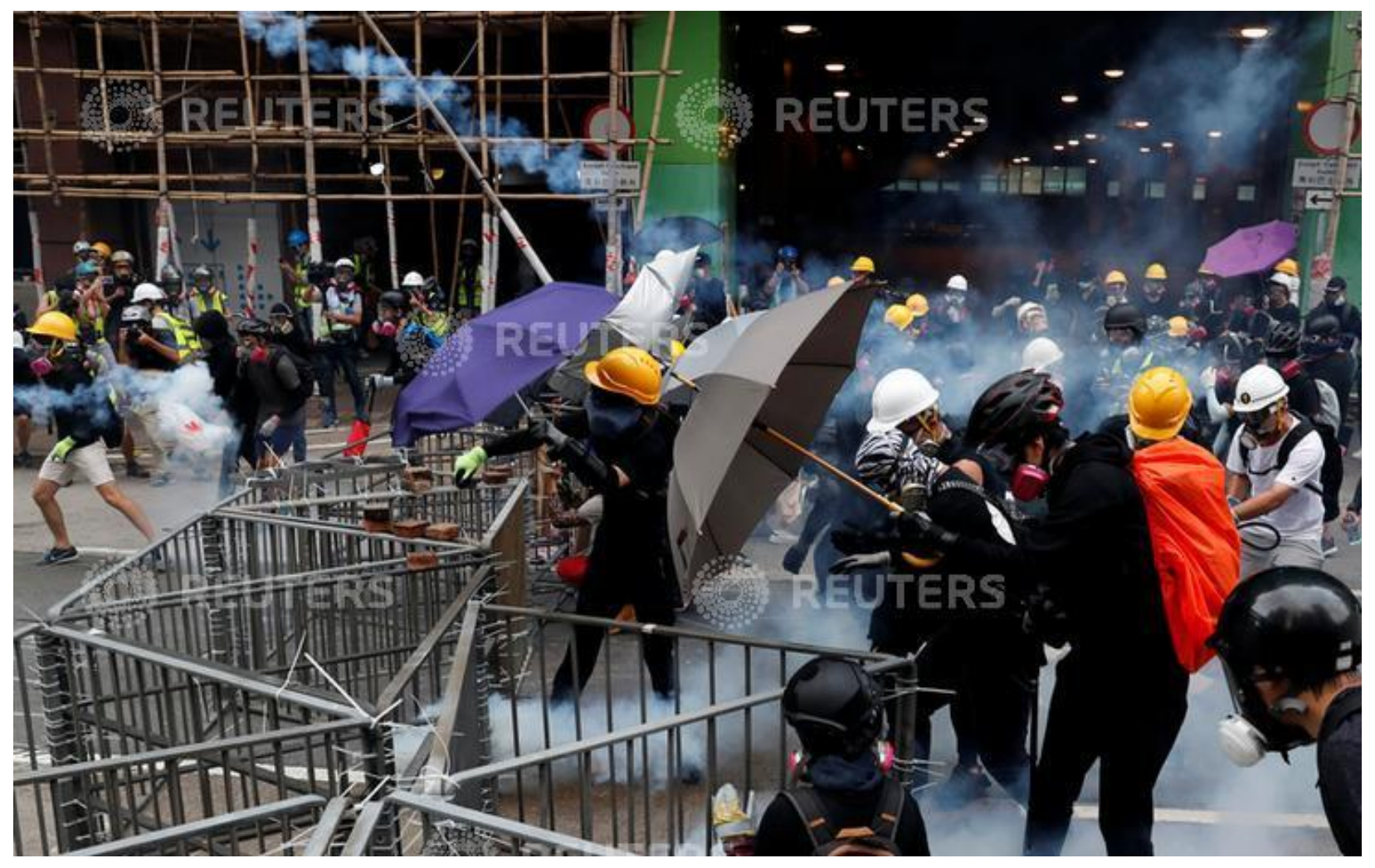

Photograph 1.3. Description from Reuters: "Anti-extradition bill protesters react after police fired tear gas during a demonstration in Sham Shui Po neighbourhood in Hong Kong, China, August 11, 2019.”

In the third photograph regarding umbrellas, we observe another chaotic situation, where protesters make use of umbrellas. We also see tear gas trails in the air above and among the protesters.

Here, we can identify five primary actors. The umbrellas. The protesters. The gas masks they are wearing. The metal barricade in front of the protesters. And lastly, tear gas. 


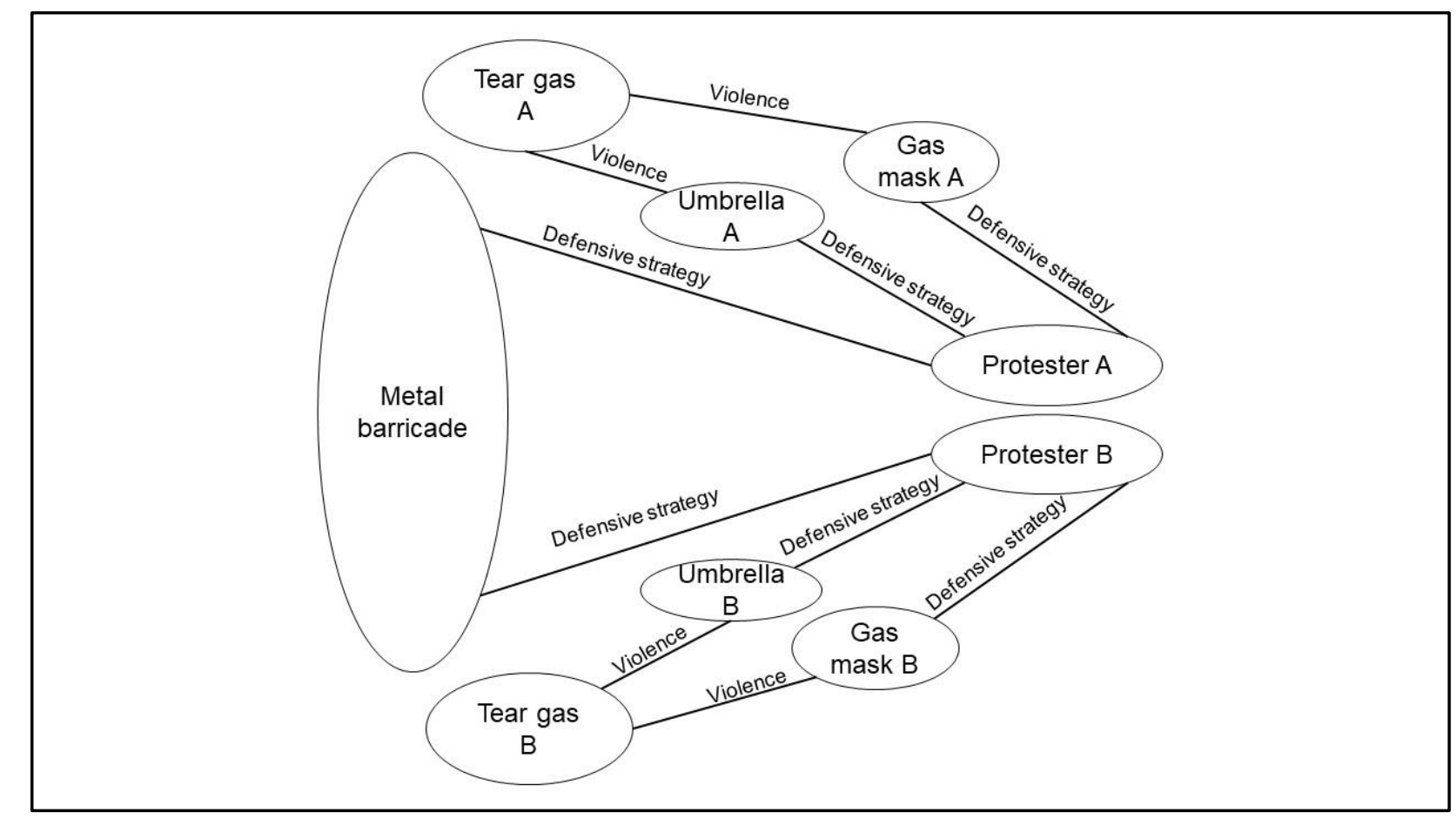

Figure 1.3. Network analysis of photograph 1.3

In this scenario, the umbrellas are again connected to the protesters via defensive strategies. On the other hand, this time the violence that the umbrellas are connected to, is the tear gas attack they shield the protesters from. At the same time, the tear gas connects to the gas masks, again through violence, while the masks themselves connect to the protesters by protection. By the same notion, the barricade connects to the protesters via yet another defensive strategy. 


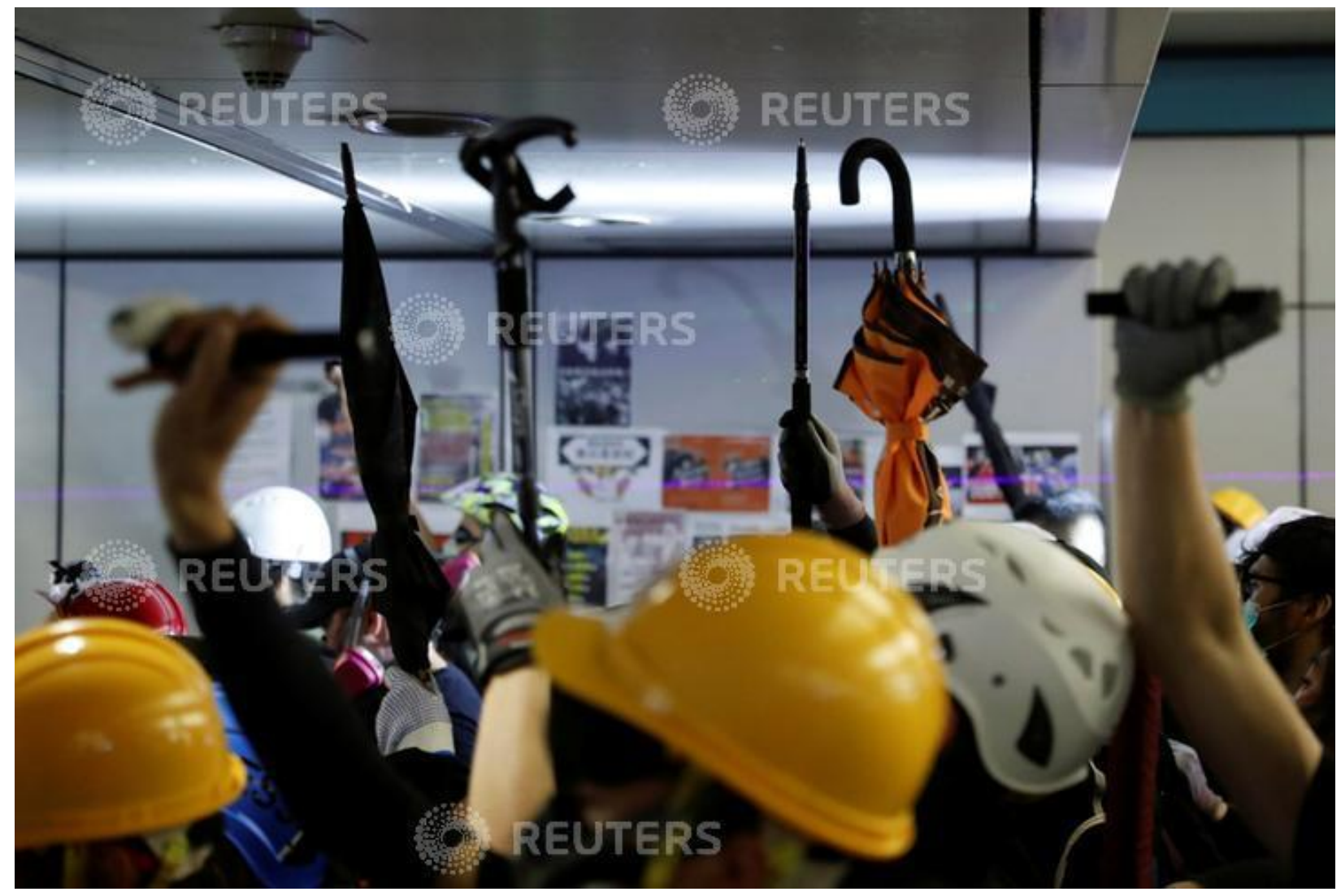

Photograph 1.4. Description from Reuters: "Protesters beat the ceiling as they face riot police during a stand off at Yuen Long MTR station, the scene of an attack by suspected triad gang members a month ago, in Yuen Long, New Territories, Hong Kong, China August 21, 2019."

In the last photograph in this section, the circumstances are somewhat unclear, when looking at the picture alone. But from the context provided by Reuters, we learn that the protesters are beating the ceiling of a metro station with their umbrellas, while facing riot police.

Here, we can identify three primary actors. The umbrellas, and for the first time in this section, in closed form, the protesters, and finally, the ceiling. We also note laser pointers, but as we cannot tell what they are pointed towards, we cannot describe their relations. In the same vein, we observe helmets, but as we cannot decipher from the photograph what their use is, they are not included in the network model. 


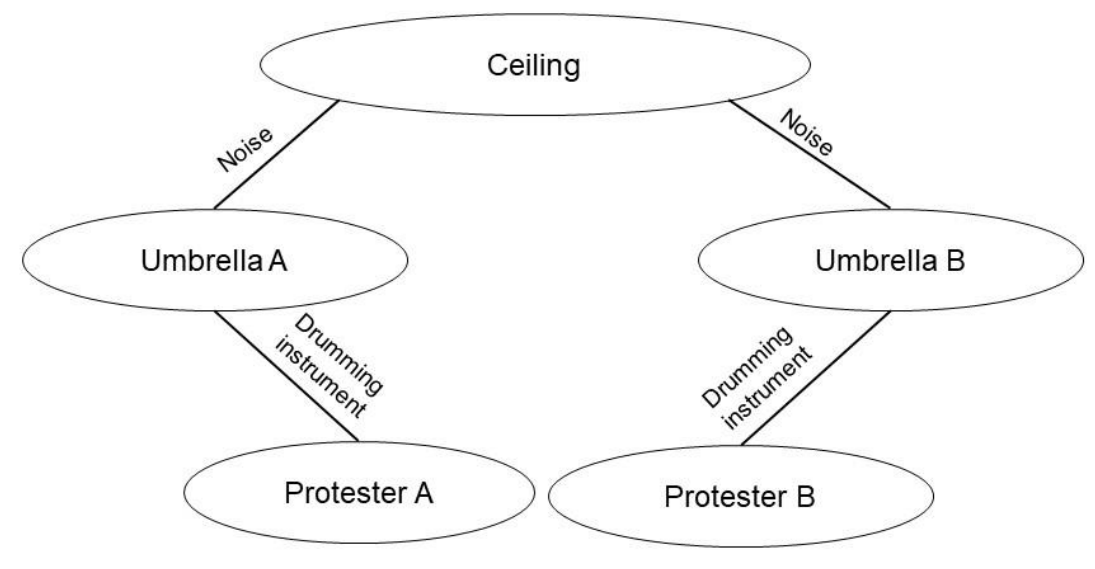

Figure 1.4. Network analysis of photograph 1.4

In this situation, the umbrellas become linked to the ceiling in terms of noise creation, and to the protesters as a drumming instrument. We see that all protesters direct their gaze in the same direction but cannot see if it is towards riot police or something else.

\subsubsection{Summary}

In the forthcoming, we will summarize the networks of the four photographs presented above into one network. This is done with the goal of summing up our analytical points as well as showing how the umbrella is translated in different situations. The connections in the summarized model are based on the connections in the smaller networks above.

In our summarized network of umbrellas, we have limited the amount of each type of actor to one in the illustration. As an example, the actor 'protesters' in the network below, thus reference more than one actor - all the protesters in our above photographical networks. 


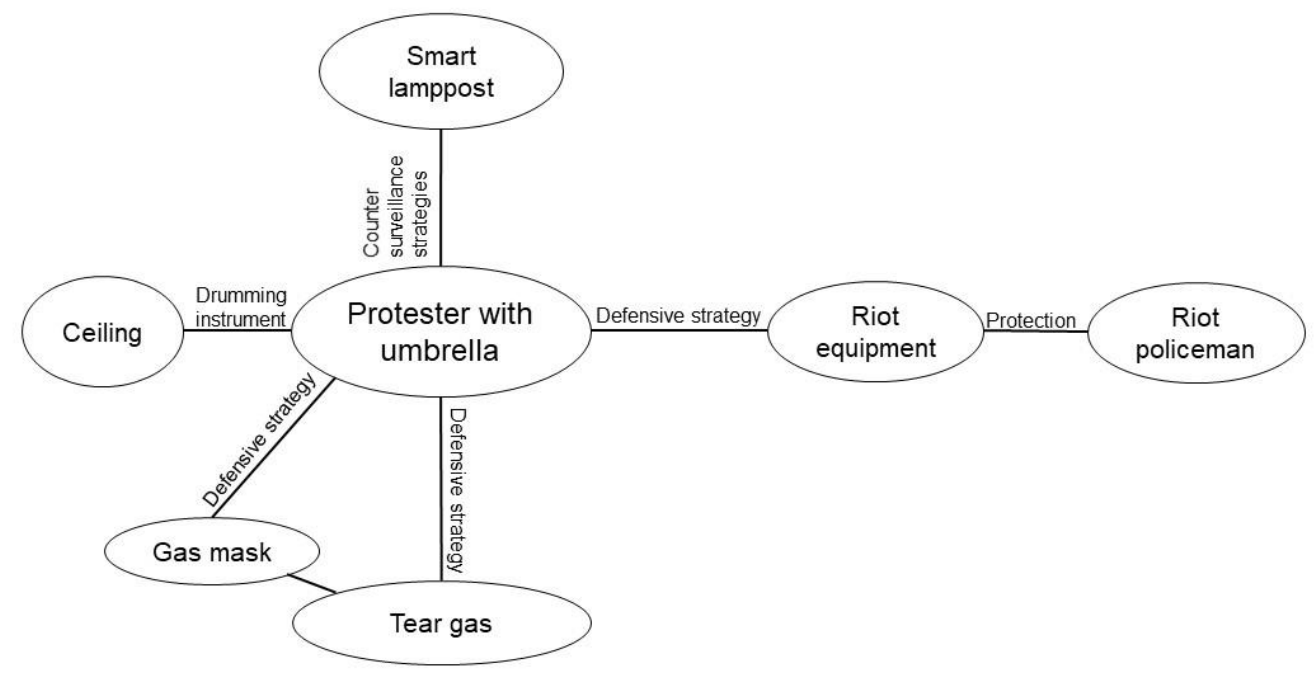

Figure 1.5. Summarizing network of the umbrella (figure 1.1, 1.2, 1.3 and 1.4).

As we observe the above network mapping, the type of link between the protester with the umbrella (here pictured as one actor for illustrative purposes) translates based upon the actor to which it is connected. As Callon notes, actor A translates actor B (Callon 1998:143). On one hand, when the umbrella (actor B) is connected to the police's riot equipment (actor A) and the tear gas, it translates into a means of defensive strategies by protecting the protest actor from violence. On the other hand, when the umbrella meets the potential gaze of the smart lamppost, it translates into a tool for counter-surveillance, by disguising the faces of protesters. And lastly, when used to bang the ceiling of a metro station, the umbrella takes the form of a drumming instrument. This shows that there is no essence to the umbrella as an actor, but rather that its meaning is constructed in its relation to another actor in the network.

\subsection{Laser pointers}

In this section of the analysis, we will look into the networks of a second non-human actor identified in the material: laser pointers.

Through network analysis of three photographs, where the laser pointers are used, we will map out their relations, and in that way show different uses of laser pointers. 


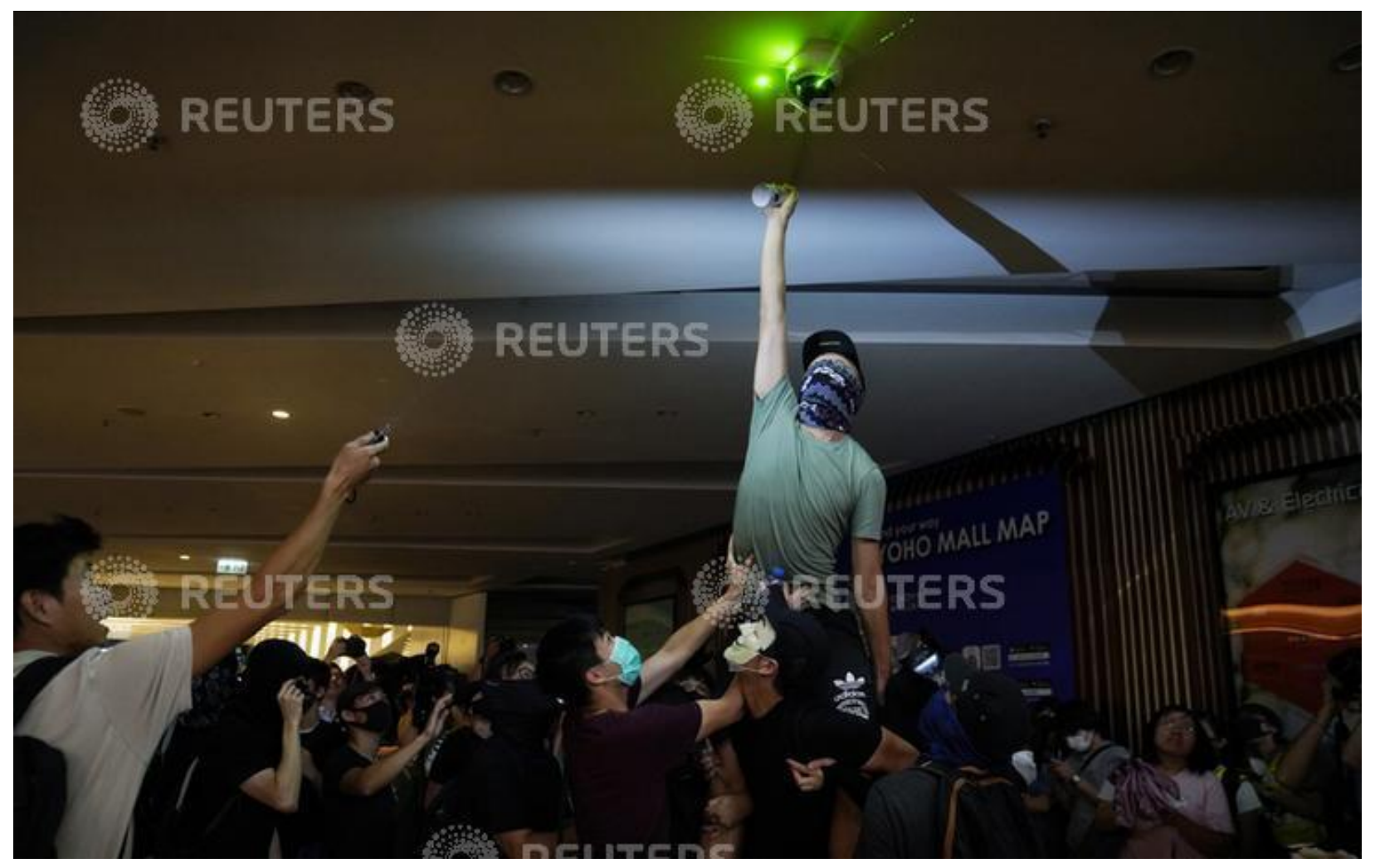

Photograph 2.1. Description from Reuters: "An anti-government protester spray-paints a security camera at Yoho Mall in Tuen Mun, Hong Kong, China September 21, 2019.”

In the first photograph, we see protesters spray-painting a security camera as well as pointing lasers directly into its lens.

We can identify four primary actors in this photograph. Firstly, the protesters. Secondly, the spray paint itself. Thirdly, the security camera. And lastly, laser pointers and the green light they emit.

Laser pointers have been used by Hong Kong protesters as a way to blind both policemen and security cameras (Bostock 2019-08-01). When the laser light is emitted into the sensors of cameras, it obfuscates its gaze upon protesters. This use has led to local authorities to refer to these laser pointers as offensive weapons, or 'laser guns' (Low 2019-08-08). 


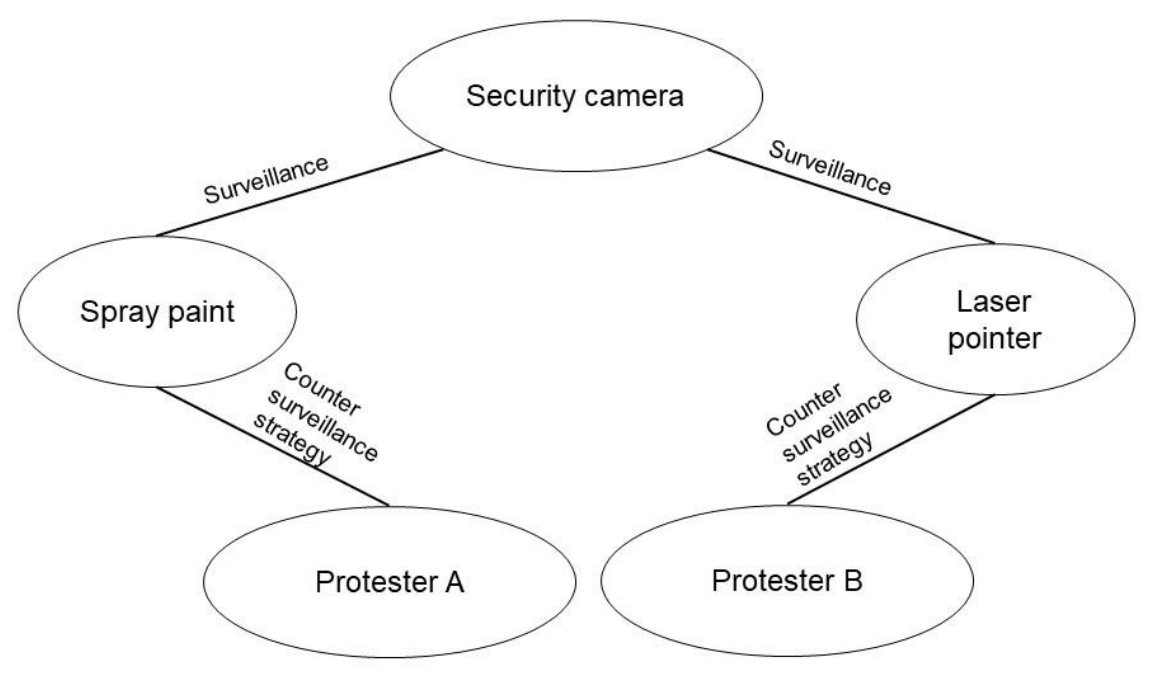

Figure 2.1. Network analysis of photograph 2.1

The security camera becomes linked to both the laser pointer and the spray paint in terms of surveillance. The two protesters translate these actors, which did not originally have this purpose, into tools for counter-surveillance strategies. The type of counter-surveillance strategy deployed here is attack, as both the laser pointer and spray paint are utilized to render the surveillance device inoperable. We see how these non-human actors influence the relation between government surveillance tactics and the protesters' counter-surveillance strategies. 


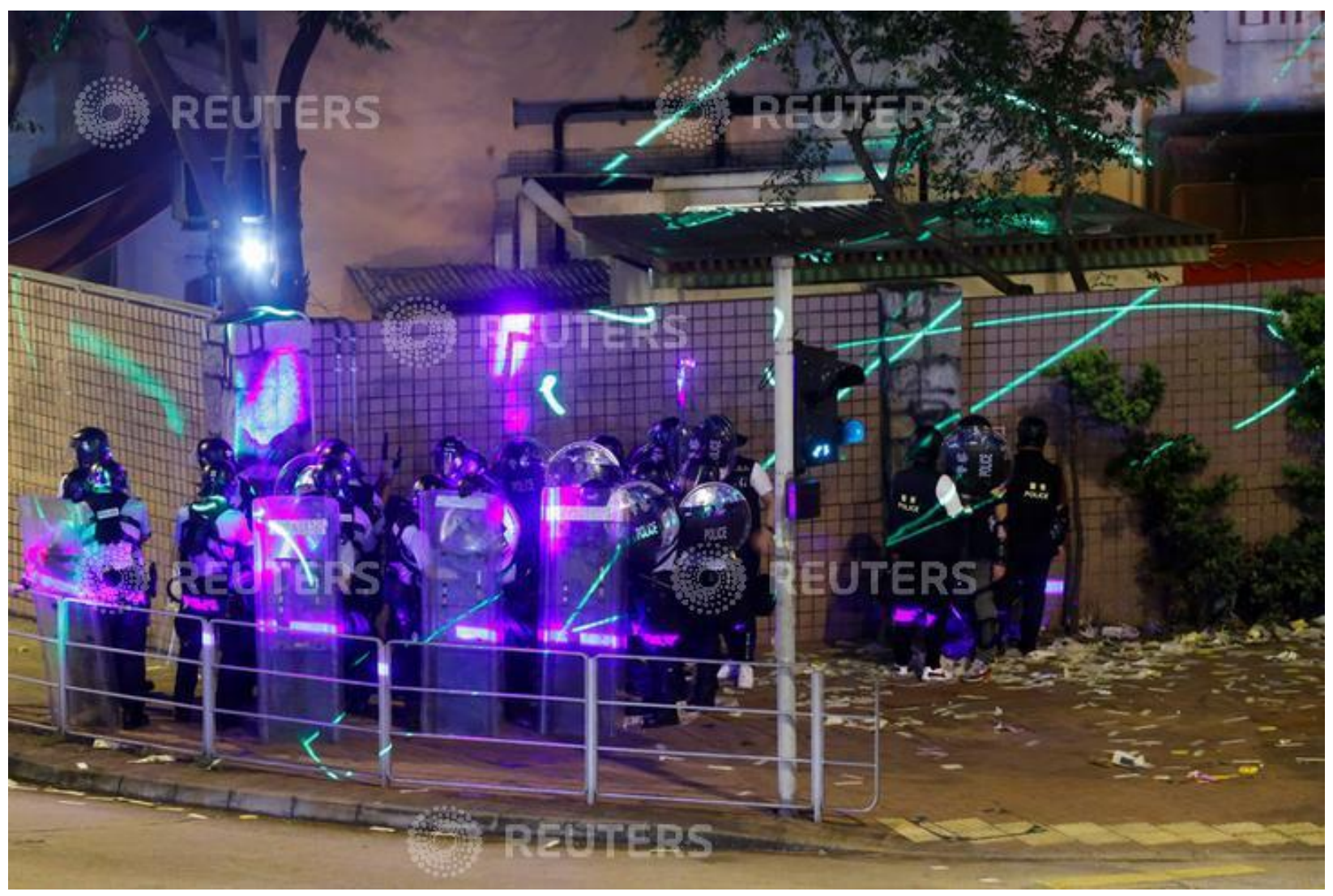

Photograph 2.2. Description from Reuters: "Anti-extradition bill protesters tease riot police with laser pens during a gathering marking Hungry Ghost Festival at Wong Tai Sin in Hong Kong, China August 9, 2019. Picture taken August 9, 2019."

In this photograph there are no protesters present in the frame. Instead, we observe multiple riot policemen and beams of purple and turquoise light flickering among them.

We can identify three actors in the above picture. Firstly, the riot policemen. Secondly, the riot equipment in their hands. Thirdly, the light emitted from multiple laser pointers.

Several of the light beams are directly pointed to the policemen at head level. This suggests again that the laser pointers are used as a blinding strategy by the protesters. However, we also see beams that are not directed at the policemen, but rather surrounds them. 


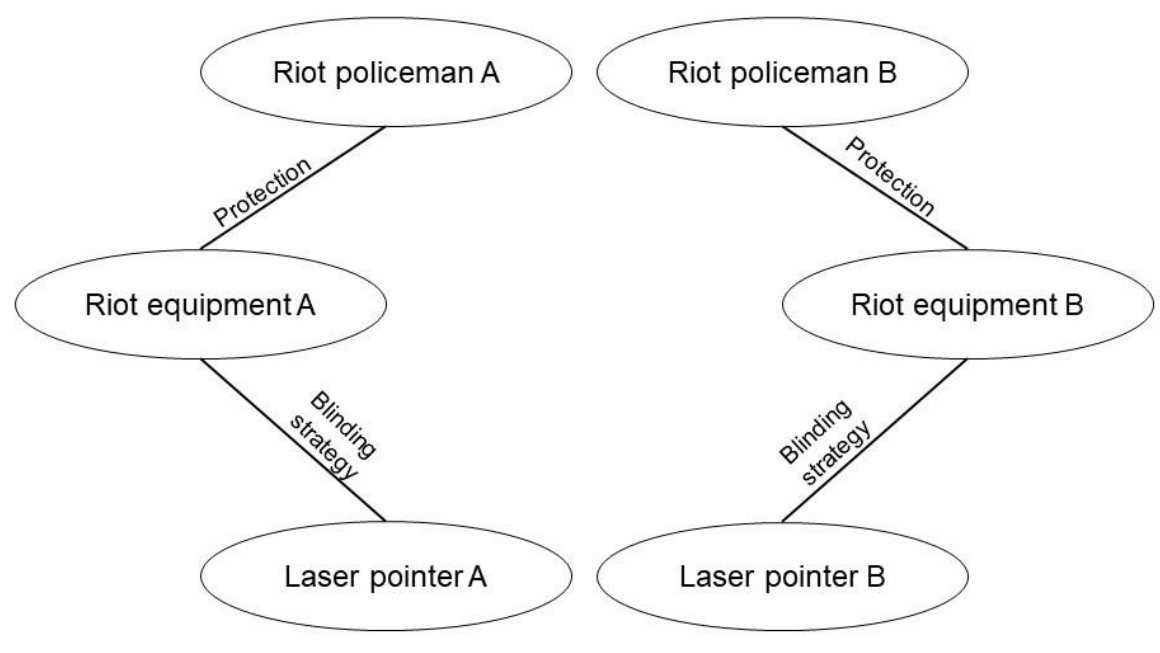

Figure 2.2. Network analysis of photograph 2.2

In this case, the laser pointers act as a strategic blinding force. Contrasting this is the riot equipment, which is linked to the policemen in terms of protection. While the laser pointers in photograph 1.1 was used as a counter-surveillance tactic towards non-human actors, we here observe it used towards human actors. 


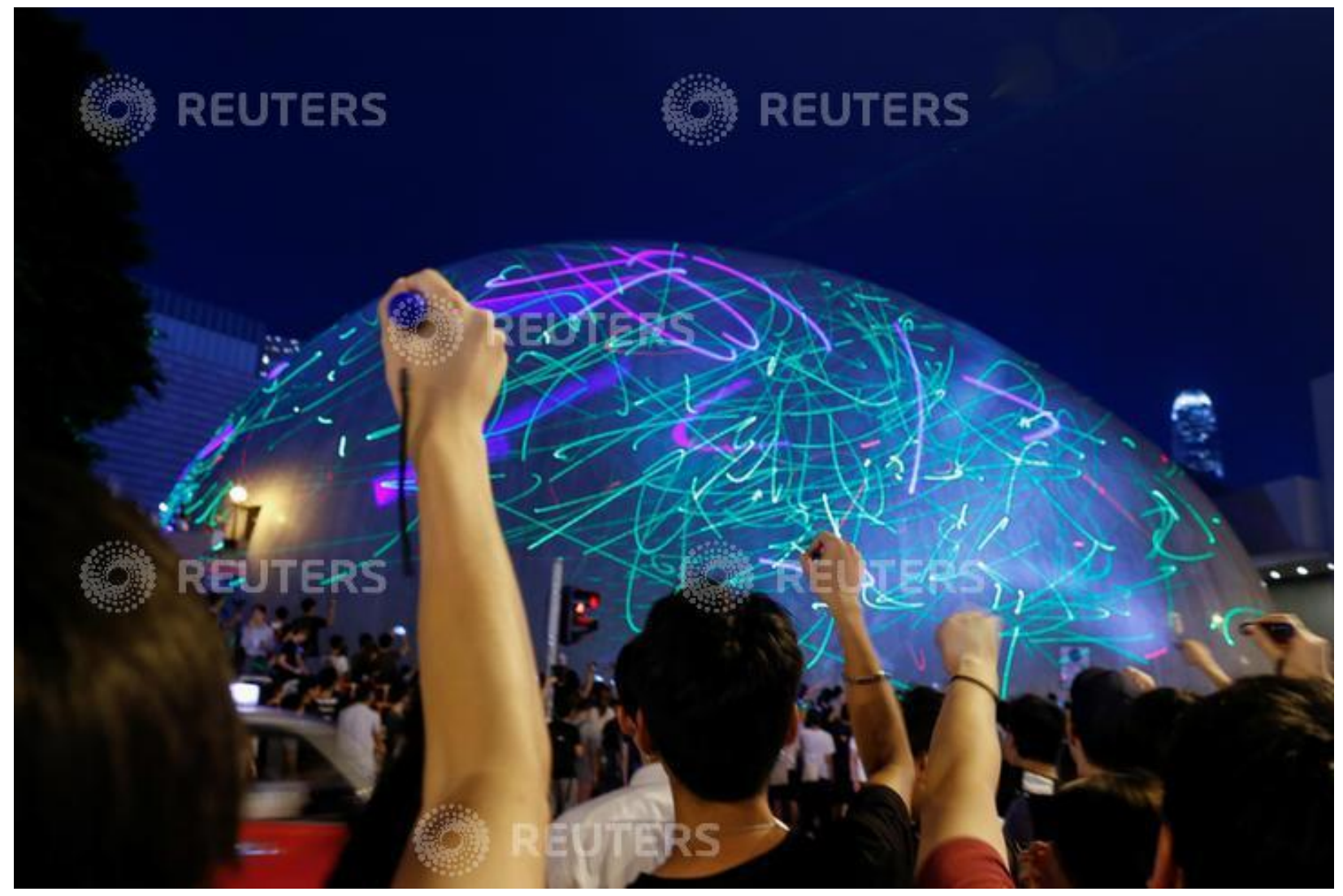

Photograph 2.3. Description from Reuters: "People watch the dots of laser pointers move across the facade of the Hong Kong Space Museum during a flashmob staged to denounce the authorities' claim that laser pointers were offensive weapons in Hong Kong, China August 7, 2019. Picture taken with a slow shutter."

In the third photography, we see protesters pointing laser pointers towards the facade of the Space Museum.

We can identify three different actors in the photograph. Firstly, the protesters, which there are multiple of in this situation. Secondly, their laser pointers, of which there are as many as there are protesters. Thirdly, the museum facade, which the laser pointers are targeted at.

The museum facade is used as a form of canvas, on which the protesters are painting with the laser pointers. All the protesters are facing the facade and targeting it in the same manner, which implies a shared or collective type of action. This also underlines the fact that the visuality of the laser pointers is strengthened, when the number of laser pointers is increased. 


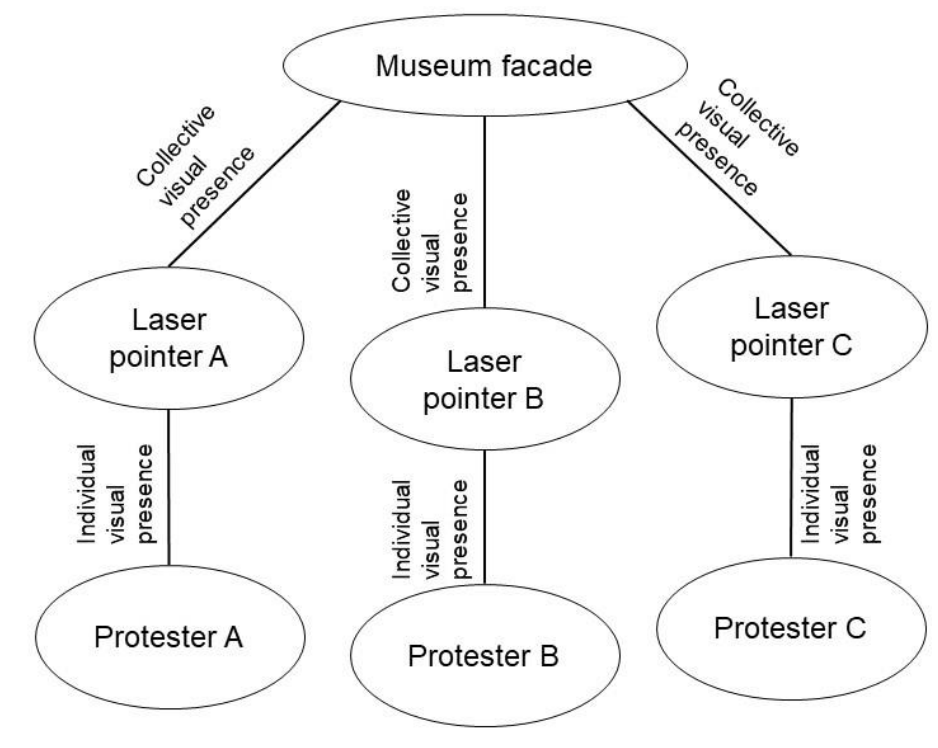

Figure 2.3. Network analysis of photograph 2.3

In this case, the protesters are linked with the laser pointers by means of an individual visual presence, whereas the laser pointers are linked with the museum facade in terms of a collective visual presence.

We see how the laser pointers, through their relations to respectively a protester on one side and the museum facade on the other side, are translated into a medium of collectivity.

In the two other figures mapping out the networks of laser pointers, we saw how they were translated into firstly a tool for performing counter-surveillance strategies, and secondly a tool for performing blinding strategies. Thus, we see a third translation of the laser pointers in this case, as they are used for the visuality itself, and not with the purpose of damaging or delimiting the nonhuman or human actors, they are targeting.

\subsubsection{Summary}

In the forthcoming, we will summarize the above networks of the three photographs into one network. This is done with the goal of summing up our analytical points, as well as showing how the laser pointer is dependent on the actors it is related to. The connections in the summarized model are based on the connections in the smaller networks above. 


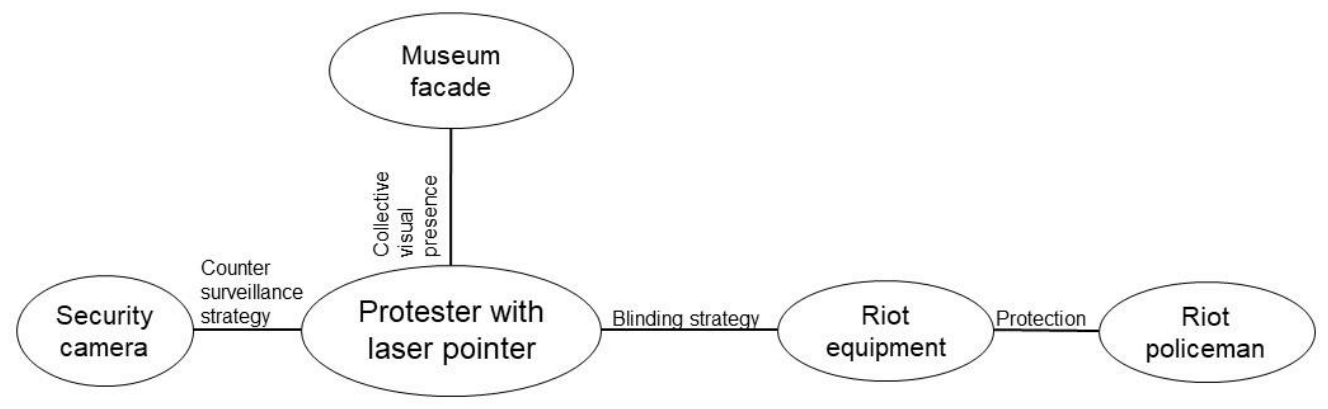

Figure 2.4. Summarizing network analysis of the laser pointer (figure 2.1, 2.2 and 2.3).

Like the umbrellas, the laser pointer's role in the networks become translated based on the actor that it is linked to. When the laser light is related to the lens of the security camera, the laser pointer is translated into a tool utilized for counter-surveillance strategies. Furthermore, when the laser pointers meet the policemen's riot equipment, the laser light is translated into a blinding strategy.

These two translations share a common notion: the light is utilized in terms of both blinding and obscuring vision. However, we believe the two translations do have differences, as the blinding strategy is oriented towards the situation the protester finds herself in. Here, avoiding confrontation and arrest of riot police. On the contrary, the counter-surveillance strategy might be thought of as a more future-oriented strategy, sabotaging surveillance technology to avoid detection by authorities, after the protest situation.

When the laser pointer meets the museum facade it translates into a different kind of actor. Here the goal is not obfuscation, sabotage nor avoidance. Instead, it is used as a way to establish a collective visual presence, without any apparent functionality beyond that. Here, the laser light becomes a visualization of the mass. A mass that becomes connected by the presence of this nonhuman actor and its visibility.

\subsection{Spray paint}

In the last section of the analysis, we will look into the networks of a third non-human actor identified in the material: spray paint. 
Through network analysis of two photographs, where spray paint is utilized, we will map out their relations, and in that way show how the spray paint is translated as a result of their relations within a network of a protest situation.

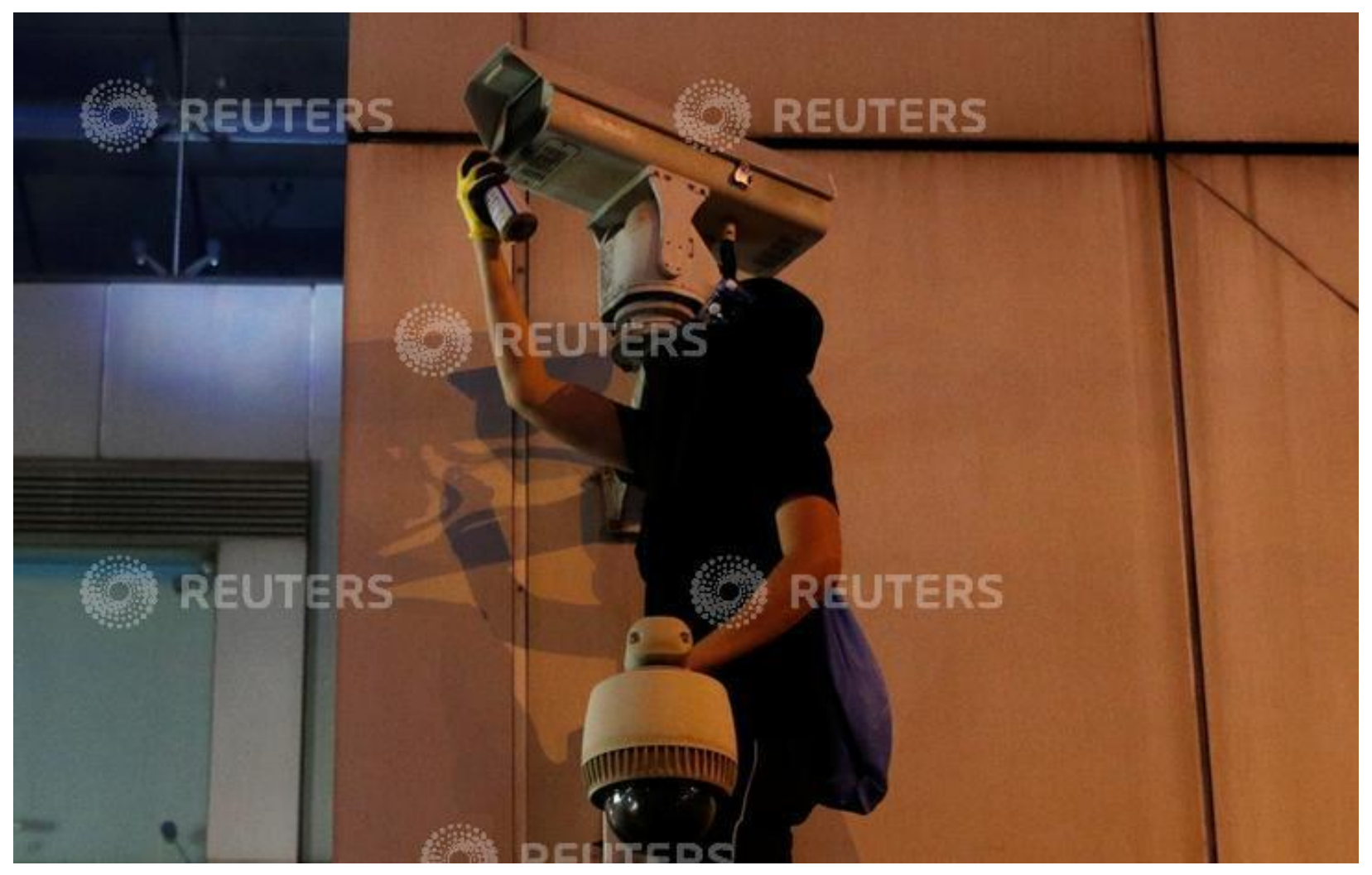

Photograph 3.1. Description from Reuters: "A protester sprays CCTV camera outside the Chinese liaison office after a march to call for democratic reforms, in Hong Kong, China July 21, 2019."

In the above picture, we observe a protester underneath a security camera. The person appears to apply spray paint to the camera lens.

Three actors can be observed in the above. Firstly, the protester. Secondly, the spray paint can in his hand and the spray paint emerging from it. Thirdly, the security camera.

As we have seen in the first two sections of the analysis, security cameras like the above one is prevalent in photographs of the Hong Kong protesters, when they engage with non-human actors. One way the protesters engage with these cameras in some of the photographs, is exactly spray painting their lenses. Several newspapers also report this activity (Bostock 2019-08-01, Dana 2019-11-13, Novak 2019-10-04). 


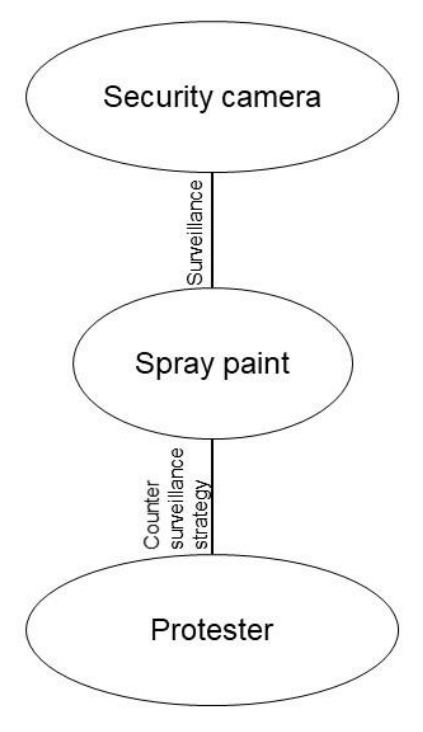

Figure 3.1. Network analysis of photograph 3.1

In this network, we again see one of the central non-human actors linked between a protester and a given actor. Here, the protester's use of spray paint translates it into a counter-surveillance strategy, as it is used to attack the security camera. In other words, the spray paint interrupts the link of surveillance that could have existed between the protester and the security camera in the above network, had it not been present in the situation. 


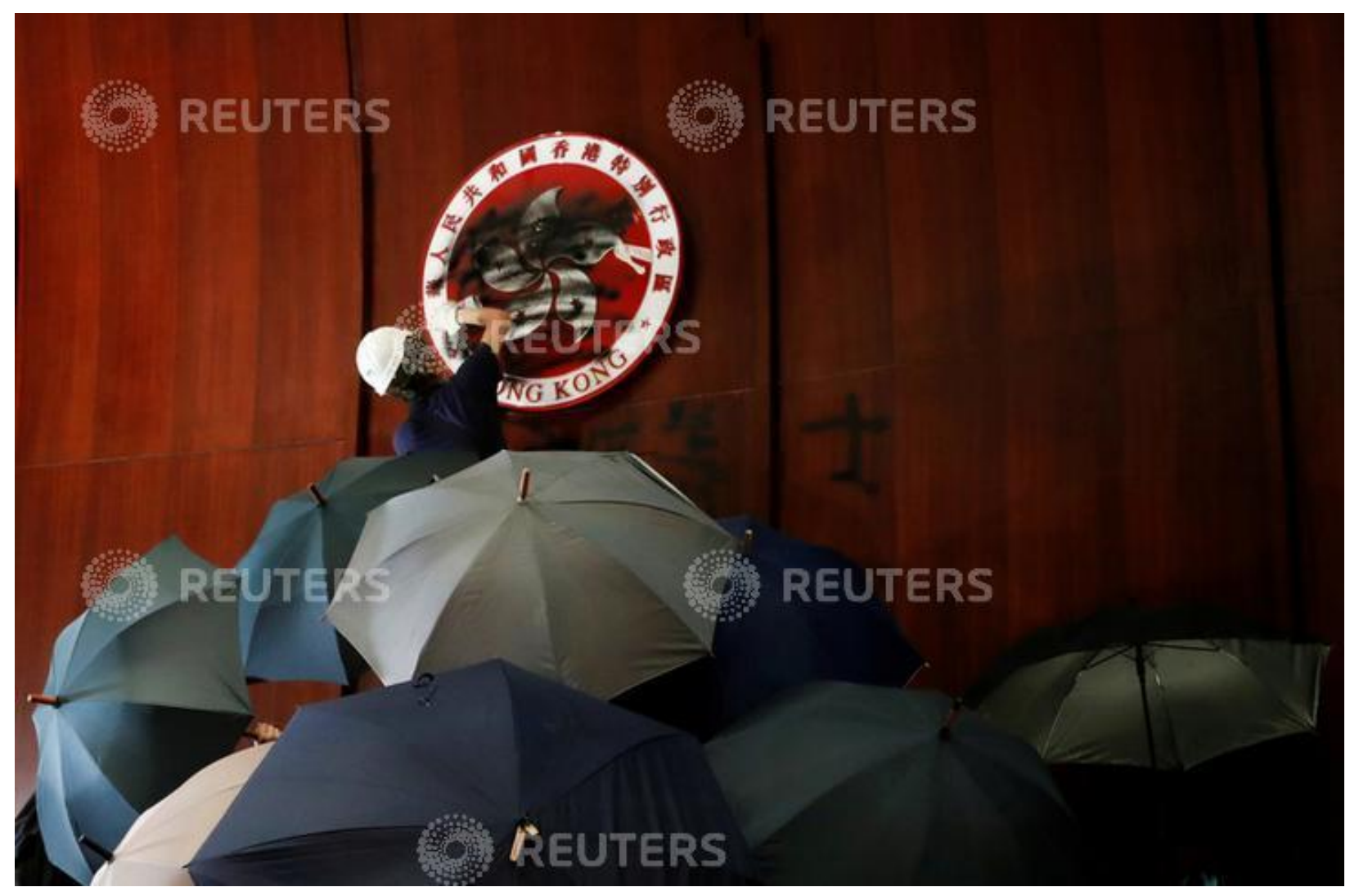

Photograph 3.2. Description from Reuters: "A person sprays paint over Hong Kong's coats of arms inside a chamber after protesters broke into the Legislative Council building during the anniversary of Hong Kong's handover to China in Hong Kong, China July 1, 2019.”

In this picture, we see protesters that have broken into the building of the Legislative Council of Hong Kong.

While one protester is doing graffiti by spray painting over the emblem of Hong Kong, the rest of the protesters screen both themselves as well as the protester doing the graffiti with umbrellas. The emblem has also been a central part of the current flag of Hong Kong since the establishment of the "One state, two systems" agreements between Britain and China in 1997 (Protocol Division Government Secretariat 2019-10-01). Thus, making it a symbol of Chinese involvement in Hong Kong.

Four actors can be identified in this photography. Firstly, the emblem, which is the target of the graffiti. Secondly, the spray paint, which is the prerequisite for making the graffiti possible. Thirdly, the protesters, who are either doing graffiti, or screening the protester doing it. And fourthly, the umbrellas, which are the prerequisite for the screening possible. 


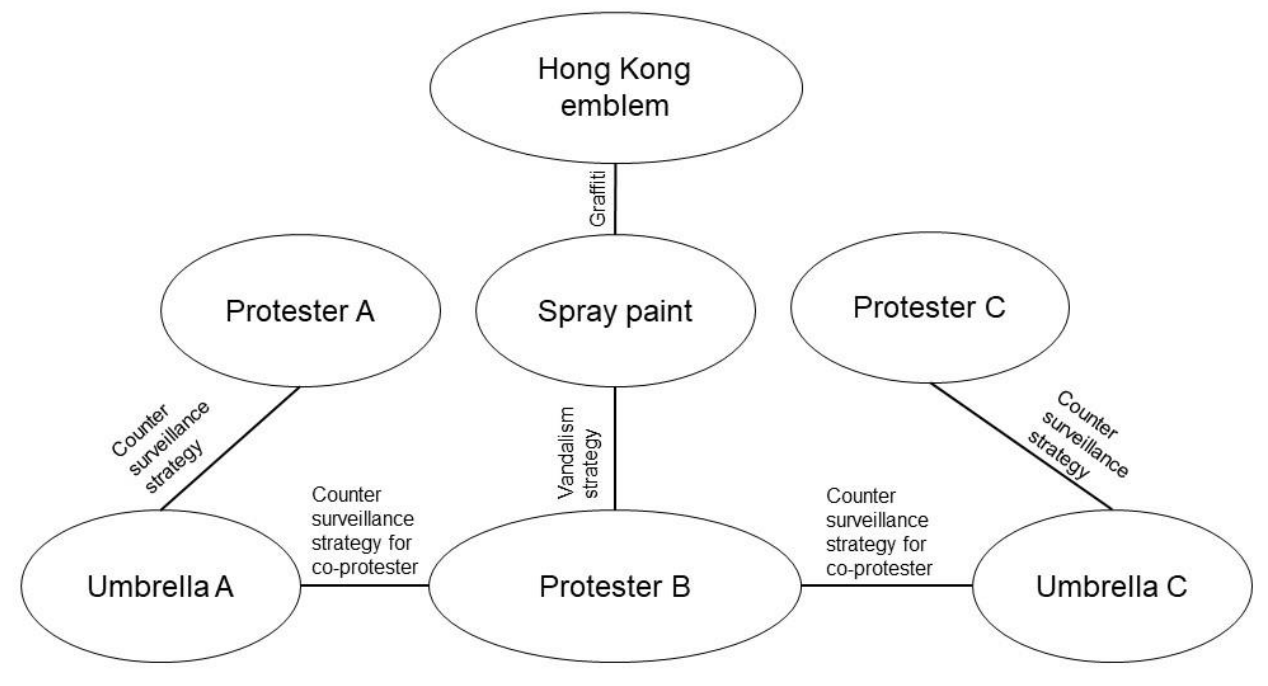

Figure 3.2. Network analysis of photograph 3.2

In the network above, we see how the non-human actors are translated within the relation to a specific protester in a specific situation. Here, protester B's use of the spray paint translates it into a vandalism strategy in order to express a political message - in this case the rejection of mainland China's influence on Hong Kong. Furthermore, we see the umbrella translated into an actor that works as a counter-surveillance strategy, both for the ones holding the umbrellas, and for the protester doing graffiti.

\subsubsection{Summary}

As we have also done in the first two sections of the analysis, we will summarize the above two networks of the photographs into one. This is done to compare different translations of the spray paint in different situations. Again, the spray paint and the protesters are portrayed as one actor for illustrative purposes. 


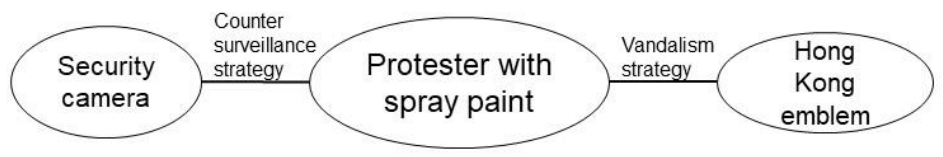

Figure 3.3. Summarizing network analysis of the spray paint (figure 3.1 and 3.2).

On one hand, when the spray paint is connected to the Hong Kong emblem, the spray paint is translated into a tool for a narrative through vandalism, as shown in the case of protesters in the Legislative Council. On the other hand, when the spray paint is connected to the security camera, it translates not into a narrative tool, but rather into a tool for conducting counter-surveillance strategies, by making the lens of security cameras opaque.

\subsection{Synthesized Network Model}

In the following section, we will outline a synthetic network model that is based on the entirety of networks in the analysis. Here, the goal is not to get at a complete mapping of the protest network. Rather, the ordeal is to try and map out a synthetic network based on the three summarizing networks, in order to get a picture of the types of relations among actors that are most prevalent in our analysis. Here is the result of this mapping: 


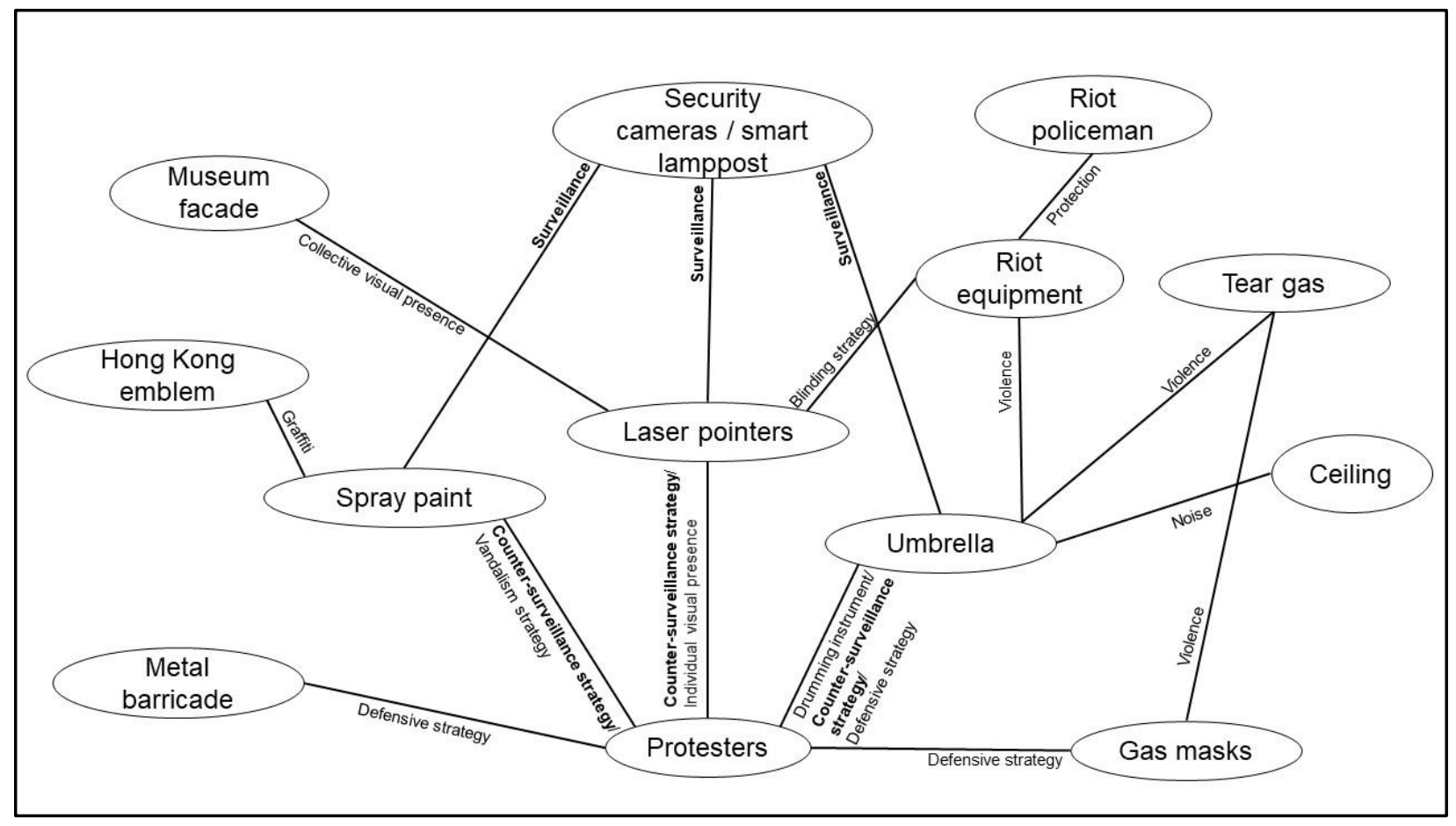

Figure 4.1. Summarizing network analysis of the umbrella, the laser pointer and the spray paint (figure 1.5, 2.4 and 3.3).

In order to understand the type of relation that seems to specifically characterize the fragmented networks that emerge from the photographs of the Hong Kong protests, we will now turn to compare the three non-human actors that our analysis has been built around, and how their translations differ and compare in relation to other actors.

We abide by the logic that if the types of relation an actor has with other actors take the same form, rather than differentiates, this actor must bear a structuring force upon relations within a network. So, to begin, we will count the types of relations that are most prevalent in the above network. In this competition relations of surveillance and counter-surveillance shares the 1st place.

The next step is to look at the actors on both ends of these surveillance and counter-surveillance relations. Here, we see that the three actors our analysis is built around - umbrella, laser pointer and spray paint - have this type of relation to other actors. We already anticipated this, but the interesting aspect is what they have in common.

For one, they are all connected to protesters in the network. This makes sense, because our selection criteria for picking out photographs from Reuters were also based on protesters being present. So analytically, this may not be too interesting. What is more striking, is that all three actors are connected to security cameras - here, the smart lamppost is also understood as a security 
camera, because the protesters treat it as such - through a relation of surveillance. In fact, it is the only actor, other than protesters, they are all linked to.

This leads us to believe that the security cameras are central actors in the protest networks and have structuring effects on how the networks are organized. Therefore, we will discuss the role of surveillance in our discussion, and how we can understand the counter-surveillance strategies deployed in relation to the security cameras. These strategies take the form of disguise tactics, when the umbrellas are translated as such, and as attacking moves, when laser pointers and spray paint is utilized. A particular point to note here, is that the non-human actors (umbrellas, laser pointers and spray paint) all intervene the surveillance relation that could have existed between the protesters and the security cameras, had they not been present and acting in the situations. 


\section{Discussion}

In our analysis, we have shown that actors in the protests translate due to their position in the network. Based on this, we can also see certain actors, most dominantly the security cameras, have a prevalent role in the relation, they engage in. This leads us to believe that surveillance plays a central role in the actor-network of the protests. In the following section, we will therefore discuss the role of surveillance and counter-surveillance strategies in the protests, and their ties to the visual dimensions of social movements. Afterwards, we will discuss the implications for social movement theory and the potential for a material turn in the literature.

\subsection{Visuality of counter-surveillance strategies}

As we have seen in the ANT analysis, the element of surveillance, as well as counter-surveillance strategies, were shown to be especially widespread relations, when we mapped the networks of the photographs. Therefore, we will initiate the following discussion based on this finding. Hereafter, we will discuss how these concepts relate to social movements.

As shown in the analysis, the Hong Kong protesters make use of what Ullrich \& Knopp call counter-surveillance strategies (2018). One such strategy is to attack, which is present in our analysis. Examples of such are protesters sabotaging smart lampposts, as well as spray painting the lenses of security cameras. Another counter-surveillance strategy is disguise, which we also find relevant in the case of Hong Kong, as they utilize umbrellas in defensive ways to render the protesters un-identifiable for local authorities.

These counter-surveillance strategies might be understood in different ways than merely a strategy to avoid surveillance. When the protesters engage in these counter-surveillance strategies, they often do so in explicit and visual ways. Instead of solely attacking security cameras under cover of the dark night, and disguising themselves by using surgical masks, they also do it in broad daylight, and with objects that emit vibrant colorful light.

Therefore, we will outline in the first part of the discussion, how the issue of surveillance and counter-surveillance in the Hong Kong protests, is not only about these two concepts in themselves - but also about visualization of a problem and a social movement.

\subsubsection{Counter-surveillance as a problematization tactic}

In the analysis, we have shown how the Hong Kong protesters engage in relations of countersurveillance strategies, by trying to sabotage and avoid surveillance. In other words, the protesters engage in what Mann calls reflectionism.

According to Mann, a key point about the conduct of reflectionism is its ability to make surveillance visible, where it would otherwise be an invisible force, often not detected by its subjects (Mann 2003:337). Were the protesters not engaging in counter-surveillance strategies, it 
is not certain that we would decipher the presence of surveillance from the photographs. When the protester holds up her umbrella towards her face, when she sabotages the smart lamppost, and when she points her laser pointer towards the security camera, surveillance becomes visible. Following Doerr, this act of creating visibility is constituting the social movement as a presence in society, while tying the issue of surveillance to the movement itself (Doerr 2013:xv). In other words, government surveillance becomes an object of problematization due to the protesters' explication of its presence, through their visual method of countering it.

One possible explanation to why the protesters specifically target government surveillance tactics, is that the security camera, to the protesters, might be seen as the incarnation of what mainland China represents: lack of democratic rights, authoritarian rule, its infiltration of the Hong Kong territory and limitations of freedom rights. In this way, the security camera becomes a proxy representation of mainland China that the protesters can physically address, sabotage and attack. The construction of this proxy makes it possible for the protesters to hit Beijing, even though it is approximately 2000 kilometers from Hong Kong. In spite of the distance, this explicitly visual conduct of counter-surveillance becomes a powerful way to problematize Chinese influence in Hong Kong matters, thus framing it as the issue that the social movement is addressing.

\subsubsection{Counter-surveillance as visual expression}

Another point following the concepts from Doerr, is related to the notion of visual expression. This will be discussed in relation to the Hong Kong protesters in the following section.

In our material from the Hong Kong demonstrations, we see how multiple protesters wear the same "uniform", or at least carry the same tools. For Doerr, the most central point of this is for the individual protester to express affiliation to the movement (Doerr 2013:xiv). In the Hong Kong case, the expressive trademarks of the movement that continuously emerge in the material, is primarily those addressed in the analysis: laser pointers, umbrellas, spray paint. Furthermore, and also present in the material, eye patches can be categorized in the same manner. At first glance, these objects (or non-human actors) serve the same function as other tools, clothes, or icons would do in other social movements. For example, red flags in socialist movements.

Yet, something is different in the case of Hong Kong, when we look at the visual expressions. Even though the umbrellas, laser pointers and spray paint have become part of what Doerr means by visual expression of the protesters, they are not entirely similar to those we know from other movements. The reason is that in Hong Kong, the visual expression is performed with objects that we identified as central to the counter-surveillance strategies. Consequently, this means that these objects do not only serve the function of visual expression but have a practical function as well. The practical function is also the primary, whereas the visual expression instead becomes an outcome of the practical function. 
The relation between the visual expression of the protesters and the practical function behind it, further consolidates that surveillance and counter-surveillance play the roles as main characters in the Hong Kong protests. In that manner, the visual expressions are entangled with the countersurveillance strategies, we mapped in the ANT analysis. Therefore, objects for performing countersurveillance become the visual expression of the Hong Kong protesters' social movement.

Following the point from the previous section regarding problematization, where countersurveillance strategies become a symbol of the protesters' discontent with mainland China, the umbrella, laser pointer and spray paint then becomes symbols of the same discontent. Or said in another way: if you are using a laser pointer in Hong Kong these days, you are opposing mainland China's interest as well as ideology. That is regardless of if the laser pointer is used to damage the sensors of a security camera (photograph 2.1), or if it is used to create a collective visual expression at a museum facade (photograph 2.3).

\subsubsection{Counter-surveillance as a visual marker}

However, the visual counter-surveillance tactics deployed are not only significant in terms of problematizing surveillance and performing visual expression. When these counter-surveillance strategies are done in very explicit and visual ways, and repeated over time, they can become what we call visual markers for a collective social movement.

Here, we will take a concrete example from our analysis to substantiate our claims: the laser pointers. To begin with, the laser pointers were translated into actors for performing countersurveillance strategies, when they were pointed towards security cameras (photograph 2.1). This utilization of laser pointers is shown in several of the photos that we have sorted from the original 5,554 photographs. In reaction to the use of laser pointers, Hong Kong local authorities held a press conference, where they termed these tools 'laser guns', and deemed them dangerous (Low 2019-08-08). Following the press conference, the protesters organized a new demonstration, where they did not use the laser pointers towards cameras, but instead pointed them towards a museum facade (photograph 2.3). Here they were used to perform a collective visual expression, suggesting that they are not offensive weapons.

This collective visualization is based on a shared utilization of laser pointers and pointing them towards the same physical object. Each tiny dot on the facade, among the many, becomes a visual representation of a collective - or in other words, a marker. As Doerr notes, a necessity for social movements is that they can constitute visual imageries to constitute themselves as actors (Doerr 2013:xv). This usage of the laser pointers is exactly one way for the social movement to establish itself through the visualization of something shared, and a way to create continuity among protesters (Doerr 2013:xiii).

The laser pointer is just one example of an actor used in counter-surveillance strategies that turns into a visual marker for the Hong Kong protests. Another example is the umbrella, which in several 
photographs are utilized both in terms of protection and as a tool for a counter-surveillance strategy. Yet, the practical usage of the umbrella is not its only purpose. Like the laser pointer, it becomes a visual marker for the social movement, because it serves as a function of expression and visibility among protesters. For the umbrella, this process has been so influential that the Umbrella-movement in 2014 got its name from its translation from practical tool to icon.

Our understanding of visual markers is then that non-human actors through chains of translation can come to gain a secondary function (other than practical) that is visual. Through further translation the secondary function can become the primary one, turning the practical quality of that actor into a new secondary function. Moreover, this increases its visual strength in the functions of visibility, expression and representation. Thus, non-human actors can become visual markers for social movements.

\subsection{Social movements in an ANT-perspective}

In this section of the discussion, we will discuss the theoretical implications of the Hong Kong case in relation to social movements. Our main argument is not that the Hong Kong protests are necessarily markedly different from other protests. Rather, it is that the broader context the protests take place within makes the influence of non-human actors more visible, which is not addressed adequately by current social movement theory. More specifically, new social movement theory.

New social movement theory (NSMT) can be seen as a response to new types of social movements emerging in the 1960s. Examples of such movements are the women's movement, the gay liberation movement and civil rights movements (Nash 2010:88). Contrary to "old" labor movements, which fought for changes in economic distribution, these movements problematized issues of for example identity (Melucci 1989:46) or citizenship rights (Nash 2010:118). In other words, new social movements were more issue-oriented than its older counterpart. We argue that the theories on these movements tend to give primacy to the immaterial in its understanding of how the issues of social movements emerge: as a social product of utterances, attitudes and human opinions.

As outlined in the above discussion section, we have looked at the role of visual markers through our ANT-based analysis and discussed how these visual markers become integral actors in Hong Kong protest networks. These visual markers are all represented by non-human actors, such as umbrellas and laser pointers, and achieve importance through their deployment in countersurveillance strategies, which is a consequence of their relations in the network.

In the first section of this discussion we have argued that counter-surveillance strategies are a way for the social movement to visually turn Chinese influence into an issue. In relation to this, we believe that the one-sided focus on immaterial human opinions and utterances in NSMT, would not have been able to encapsulate the importance of material non-human actors in forming the issue of the Hong Kong protest network. If we had utilized a more traditional NSMT-based 
perspective in this project, we would certainly have been able to identify what the issue that the Hong Kong social movement addresses is. But we would not have been able to understand the process of how this issue is situated in its relation to non-human actors present in the specific situations of the protest. More specifically, how it is formulated through the protesters' practice in the protest situation itself, and through their relation to specific non-human actors, who later translates into visual markers. Yet, like NSMT-theorists, we believe that the issue of a social movement is an integral element of its existence and actual practice. Understanding the process of how the issue is situated is therefore interesting, because it gives perspective on how a central element to organizing a social movement is the translation of non-human actors.

Our recommendation for future studies of social movements is therefore to abide by especially two ANT-mantra: 1) not to map out homogeneous networks, where human actors are prioritized over non-human actors, but instead describe associations between heterogeneous elements (Latour 2005:5), and 2) to follow the actors and their relations to expand the network (Latour 2005:12). By doing so, it will become possible for the researcher to look at situational practices, describe relations between actors, and utilizing these descriptions to understand the formulation of a social movement's issue. 


\section{Limitations and further research}

In the following, we will provide recommendations for further research on the Hong Kong protests, based on the methodological and theoretical limitations of our project framework.

One limitation of our project framework is that our focus on the actors of the protests means that we remain at the descriptive level in our analysis, and that we can only look at the microinteractions in specific situations. If we were to examine the protest from a more macro-oriented perspective, we would need to approach our analysis differently.

Should we continue our research, there could be two different paths to take: 1) strengthening our ANT analysis with more and different types of types of empirical material, and 2) taking another approach that would allow us to delve deeper into the political power relations of the controversy.

Continuing our ANT approach, we would widen our empirical material to achieve a more comprehensive analysis. One way could be to conduct fieldwork in Hong Kong and take the photographs for the analysis ourselves. This would be more in line with Latour's way of using photographical material in Pandora's Hope and Paris: Invisible City, where the photograph is part of the ANT based fieldwork output, done by a scholar present in the situation of practice, and not - as in our project - an independent piece of material collected second handedly, and unrelated to the motive it is depicting.

Taking another approach, we could start by looking into different types of text material, e.g. statements such as press releases or Twitter posts from both authorities of Hong Kong and the protesters. Furthermore, we would have considered doing qualitative interviews with representatives from both sides. By doing so, we could have added another dimension to our analysis: one that focused more on discourse.

Adding a discourse perspective, we could have looked into one of Doerr's notions that did not suit our ANT approach. According to Doerr, an important aspect for social movements is how they are represented in the media, and how it is closely related to their visuality (Doerr 2013:xiv). The representation of a social movement is not controlled by the movement itself - but is a contested area, influenced by several actors. In our case, how the Hong Kong government portrays the movement to the public and how this image is contested by protesters on social media, could be an interesting perspective. We have not touched upon in this in our project, as we have not been able to meaningfully include empirical material that could illuminate this perspective, while also going into depth with our photographical analysis.

In relation to the battle for the narrative, there is the notion of sousveillance in Mann's framework, which is describing when "individuals [are] using tools to observe the organizational observer" (Mann 2003:333). Instances of such are present in our empirical material, for example when protestors utilize mobile phones to film and collect data on local authorities. The reason for us not 
including this perspective is that it had limited presence in our material. Going forward it could be beneficial to look into this specific type of counter-surveillance strategy (Ullrich \& Knopp 2018:190). A different perspective that could be pursued, which is also related to the notion of representation mentioned above, is a 'meta-surveillance' perspective. This would take the form of a more macro-oriented analysis of how the world society is monitoring China in regard to human rights as well as the Hong Kong protests, or how commercial trading interests related to China, influence the willingness of politicians to support pro-democratic protesters in Hong Kong. 


\section{Conclusion}

In this last section of our project, we will conclude and summarize the approach and findings of our inquiry into the Hong Kong 2019 protests.

In this project, we have investigated how certain technologies and objects become central actors in networks of protests in Hong Kong across the dimensions of knowledge, organizations and politics. Our reason to deploy this perspective was based on a missing focus on materiality and non-human actors in current social movement literature.

Inspired by visual sociology and ANT, our approach to studying this development have been to look at the chains of translations that these technologies and objects have undergone in situations of protest. Concretely, we have selected 83 photographs of the Hong Kong protests from a photographical archive of 5,544 photographs gathered from the news agency Reuters, which we have based our analysis on. The strategy for analyzing the photographs was to map out the network of human and non-human actors in each photograph by looking into their micro-interactions.

Theoretically, we build upon Bruno Latour and Michel Callon's concepts from ANT. We also include Nicole Doerr's understandings of visuality in regard to social movements, as well as Steve Mann's concept of reflectionism and Peter Ullrich \& Phillipp Knopp's typologies of countersurveillance strategies.

In our analysis, we have focused on three non-human actors that we identified in the empirical material: umbrellas, laser pointers and spray paint. Our analytical findings suggest that the translations of each of these non-human actors center especially around relations of surveillance and counter-surveillance. More specifically, the way these actors are utilized, and thus translated, are often for protesters to either attack surveillance technology devices or to disguise themselves from surveillance.

We have discussed the significance of this finding in our discussion. Firstly, we pointed to how the protester's engagement in visual counter-surveillance strategies served to problematize the surveillance itself - and ultimately, Chinese influence in Hong Kong. Secondly, we argue that conducting counter-surveillance becomes a way for the individual protester to visually express her resistance. Thirdly, we argue that the non-human actors used to counter surveillance become a central visual marker for the social movement. This means that the non-human actors have translated from being practical devices to becoming tools for visual expression of a collective. Afterwards, we discussed the implications of our findings for social movement theory. In short, our suggestions are for social movement theory to take the materiality of social movements into higher consideration. 
Lastly, we presented the limitations of our project, while reflecting on what we would do to both strengthen our ANT perspective, and to investigate other aspects of the Hong Kong protests in further research. 


\section{Literature}

Altheide, David L. \& Christopher J. Schneider (2012): Qualitative Media Analysis. SAGE Publications.

Anon. (2019-10-23): "Hong Kong Scraps Law That Sparked Protests.” BBC.com, October 23, 2019. https://www.bbc.com/news/world-asia-china-50150853 (retrieved December 12, 2019).

Anon. (2019-11-28): “The Hong Kong Protests Explained in 100 and 500 Words." BBC.com, November 28, 2019. https://www.bbc.com/news/world-asia-china-49317695 (retrieved December 16, 2019).

Becker, Howard (1995): Visual sociology, documentary photography, and photojournalism: It's (almost) all a matter of context. Visual Studies 10(1-2).

Bennett, Julia (2018): "Whose Place Is This Anyway? An Actor-Network Theory Exploration of a Conservation Conflict.” Space \& Culture 21(2).

Bischoff, Paul (2019-08-15): “The World's Most-Surveilled Cities.” Comparitech.com. August 15, 2019. https://www.comparitech.com/vpn-privacy/the-worlds-most-surveilled-cities/ (retrieved December 16, 2019).

Bostock, Bill (2019-08-01): "Video shows Hong Kong protesters using lasers to disrupt government facial-recognition cameras", Businessinsider.com, August 1, 2019. https://www.businessinsider.com/video-hong-kong-protesters-point-lasers-blind-facialrecognition-cameras-2019-8?r=US\&IR=T (retrieved November 30, 2019).

Callon, Michel (1998): Techno-economic networks and irreversibility. The Sociological Review 38.

Callon, Michel \& Bruno Latour (1981): Unscrewing the big Leviathan: how actors macrostructure reality and how sociologists help them to do so, in Knorr-Cetina and Cicourel, A.V: Advances in social theory and methodology. Toward an integration of micro-and macrosociologies. Boston: Routledge and Kegan Poul.

Chan, Kin-man (2015): “Occupying Hong Kong.” Sur - International Journal on Human Rights.

Dana, Felipe (2019-11-13): "Portraits of Hong Kong's masked protesters - in pictures", Theguardian.com, November 13, 2019. https://www.theguardian.com/world/2019/nov/13/portraits-of-hong-kongs-masked-protesters-inpictures (retrieved December 12, 2019). 
Denzin, Norman K. and Yvonna S. Lincoln (2012): Strategies of Qualitative Inquiry. SAGE Publications.

Doerr, Nicole (2013): Toward a Visual Analysis of Social Movements, Conflict, and Political Mobilization. In Lisa Leitz, Research in Social Movements, Conflicts and Change. Emerald Group Publishing Limited.

Flyvbjerg, Bent (2006): Five misunderstandings about case-study research. Qualitative Inquiry 12(2).

Harper, Douglas (2012): Visual Sociology. Routledge.

Hermida, Alberto and Víctor Hernández-Santaolalla (2018): "Twitter and Video Activism as Tools for Counter-Surveillance: The Case of Social Protests in Spain.” Information, Communication \& Society 21(3).

Kaiman, Jonathan (2014-09-30): "Hong Kong's Umbrella Revolution - the Guardian Briefing." Theguardian.com, September 30 2019. https:/www.theguardian.com/world/2014/sep/30/-sphong-kong-umbrella-revolution-pro-democracy-protests (retrieved December 12 2019).

Latour, Bruno (1993): We Have Never Been Modern. Harvard University Press.

Latour, Bruno (2000): Circulating Reference. Sampling the soil in the amazon forest. In Latour, Bruno (2000): Pandora's hope - essays on the reality of science studies. Harvard University Press.

Latour, Bruno (2005): Reassembling the social: an introduction to actor-network-theory. Oxford University Press

Latour, Bruno \& Emilie Hermant (2006): Paris: Invisible City.

Leal, Eduardo (2019-08-16): "Hong Kong's Descent to Emergency Rule: 118 Days of Unrest." Bloomberg.com, August 16 2019. https://www.bloomberg.com/graphics/hong-kong-proteststimeline/ (retrieved December 9, 2019).

Leigh, Karen, Sheryl Tian Tong Lee, Chloe Whiteaker and Natalie Lung (2019-08-14): "Hong Kong's Descent to Emergency Rule: 118 Days of Unrest”, Bloomberg.com, August 14, 2019. https://www.bloomberg.com/graphics/hong-kong-protests-timeline/ (visited November 30, 2019).

Li, Jeff (2019-12-13): "Hong Kong-China Extradition Plans Explained.” Bbc.com, December 13. https://www.bbc.com/news/world-asia-china-47810723 (retrieved December 16, 2019). 
Livni, Ephrat (2019-08-24): "Hong Kong protesters are attacking smart lampposts", Qz.com, August 24, 2019. https://qz.com/1694684/hong-kong-protesters-are-attacking-smart-lampposts/ (visited November 30, 2019).

Low, Zoe (2019-08-08): "Laser pointers are in the spotlight in Hong Kong's protests and selling briskly. But are they 'offensive weapons' and can you be arrested for carrying one?", Scmp.com, August 8, 2019. https://www.scmp.com/news/hong-kong/politics/article/3021897/laser-pointersare-spotlight-hong-kongs-protests-and (retrieved November 30, 2019).

Mann, Steve, Jason Nolan \& Barry Wellman (2003): Sousveillance: Inventing and Using Wearable Computing Devices for Data Collection in Surveillance Environments. Surveillance \& Society 1(3).

Melucci, Alberto (1989). Nomads of the Present: Social Movements and Individual Needs in Contemporary Society, ed. J. Keane and P. Mier. London: Hutchinson Radius.

Novak, Matt (2019-10-04): "Hong Kong Announces Ban on Masks and Face Paint That Helps Protesters Evade Facial Recognition", Gizmodo.com, October 4, 2019.

https://gizmodo.com/hong-kong-announces-ban-on-masks-and-face-paint-that-he-1838765030 (retrieved December 12, 2019).

Pedersen, Kaare (2011): "The research problem and problem formulation". In Olsen, P. B., \& Kaare Pedersen: Problem-Oriented Project Work: a workbook. Roskilde Universitetsforlag.

Protocol Division Government Secretariat (2019-10-01): "Introduction", Protocol.gov.hk, October 1, 2019. https://www.protocol.gov.hk/flags/eng/intro/index.html (retrieved December 17, 2019).

Ragin, Charles C. (1992): "Casing" and the process of social inquiry. In Becker, Howard and Charles Ragin, What is a case? Exploring the foundations of social inquiry. Cambridge University Press.

Rodríguez-Giralt, Israel (2011): "Social Movements as Actor-Networks: Prospects for a Symmetrical Approach to Doñana's Environmentalist Protests." Convergencia: Revista de Ciencias Sociales 18(56).

Rodríguez-Giralt, Israel, Isaac Marrero-Guillamón, and Denise Milstein (2018): "Reassembling Activism, Activating Assemblages: An Introduction.” Social Movement Studies 17(3).

Savage, Mike \& Roger Burrows (2007): The coming crisis of empirical sociology. Sociology $41(5)$. 
Tai, Benny (2019): “30 Years After Tiananmen: Hong Kong Remembers.” Journal of Democracy $30(2$

Ullrich, Peter and Gina Rosa Wollinger (2011): "A Surveillance Studies Perspective on Protest Policing."

Ullrich, Peter \& Philipp Knopp (2018): Protesters' Reactions to Video Surveillance of Demonstrations: Counter-Moves, Security Cultures, and the Spiral of Surveillance and CounterSurveillance. Surveillance \& Society 16(2).

Wong, Brian and John Mak (2019-10-30): "Column: Hong Kong's Relations With China Badly Need Reform”, Time.com, October 30, 2019. https://time.com/5713715/hong-kong-one-countrytwo-systems-failure/ (retrieved December 16, 2019).

Wu, Jin, K. K. Rebecca Lai, and Alan Yuhas (2019-11-20): "Six Months of Hong Kong Protests. How Did We Get Here?” Nytimes.com, November 202019.

https://www.nytimes.com/interactive/2019/world/asia/hong-kong-protests-arc.html (retrieved 12 December 2019).

Yeo, Rachel (2019-07-17): "Hong Kong unveils plan to install 400 multifunctional lamp posts as part of smart city drive", Scmp.com, July 17, 2019. https://www.scmp.com/news/hongkong/society/article/3018877/government-unveils-plan-install-400-multifunctional-lamp (retrieved November 30, 2019). 